\title{
Screening-Off and Causal Incompleteness: A No-Go Theorem
}

\author{
Elliott Sober and Mike Steel
}

\begin{abstract}
We begin by considering two principles, each having the form causal completeness ergo screening-off. The first concerns a common cause of two or more effects; the second describes an intermediate link in a causal chain. They are logically independent of each other, each is independent of Reichenbach's principle of the common cause, and each is a consequence of the causal Markov condition. Simple examples show that causal incompleteness means that screening-off may fail to obtain. We derive a stronger result: in a rather general setting, if the composite cause $C_{1} \& C_{2} \& \ldots \& C_{n}$ screens-off one event from another, then each of the $n$ component causes $C_{1}, C_{2}, \ldots, C_{n}$ must fail to screen-off. The idea that a cause may be ordinally invariant in its impact on different effects is defined; it plays an important role in establishing this no-go theorem. Along the way, we describe how composite and component causes can all screen-off when ordinal invariance fails. We argue that this theorem is relevant to assessing the plausibility of the two screening-off principles. The discovery of incomplete causes that screen-off is not evidence that causal completeness must engender screening-off. Formal and epistemic analogies between screening-off and determinism are discussed.
\end{abstract}

\section{Introduction}

2 Influence and Non-degeneracy Conditions

3 A No-Go Theorem for Two Dichotomous Causes and its Limitations

4 A More General No-Go Theorem: Allowing Several Causes, Possibly Nondichotomous

5 Examples Illustrating Corollary 3 and Theorem 5 a

6 Determinism and Screening-Off: Disanalogy and Analogy

Appendix 


\section{Introduction}

Screening-off gets discussed in two causal contexts. First, in a causal chain from $C$ to $I$ to $E$, there is the idea that the intermediate link $I$ screens-off $C$ from $E$. For example, when Joe dials Sally's telephone number, this causes Sally's phone to ring, and her phone's ringing causes her to pick up. In the old days, the ringing of Sally's phone screened-off Joe's dialling from her answering; given that her phone is ringing, the probability of her picking up is the same, whether or not Joe dialled her number. But times have changed. With caller-ID there now are two ways that Joe's dialling Sally can affect whether she picks up her phone; his dialling causes her phone to ring and his dialling causes her caller-ID window to say 'Joe Schmoe is calling'. Now the phone's ringing does not screen-off Joe's dialling from Sally's picking up.

The second context in which screening-off gets discussed involves a common cause that is said to screen-off its joint effects from each other. For example, in Mendelian genetics, when two parents have two offspring, the genotype of the parental pair screens-off one offspring's genotype from the other's. Given that the parental pair is $A A$ and $A a$ at a locus, the probability that one offspring is $A A$ is $1 / 2$ regardless of what the other offspring's genotype happens to be. This simple relation has not been supplanted by modern technology (at least, not yet).

When caller-ID came on line, 'the ringing of Sally's phone' ceased to provide a 'complete description' of the causally relevant events that occurred at that time. With this failure of completeness, the ringing no longer screened-off. A similar point pertains to Mendelian genetics. Although the genotype of the parental pair screens-off one offspring's genotype from the other's, the genotype of just one parent does not. Given that mom is $A A$, the probability that one offspring is $A A$ takes different values depending on what the genotype is of the other offspring. Here again, an incomplete cause fails to screen-off.

Phone calls and Mendelian reproduction are, of course, just examples; there are general principles concerning screening-off by intermediate links and by common causes. Since we wish to discuss how these principles are related to each other, we will formulate them with reference to a single set of variables. But first we need to explain our notation and define two concepts. As shown in Figure 1, the variables $E_{1}$ and $E_{2}$ at $t_{3}$ represent two effects of the proximate cause $C_{p}$ at $t_{2}$, which in turn is caused by the distal cause $C_{d}$ at $t_{1}$. Each variable can take various discrete values (we'll say that a variable is dichotomous when it has two values and nondichotomous when it has three or more); when it takes a value, the result is what we will call 'an event'. For example, ' $C_{d}=1$ ' represents the event of the variable $C_{d}$ being in state one. To philosophers wedded to the idea that causation is a relation between events, it may sound 


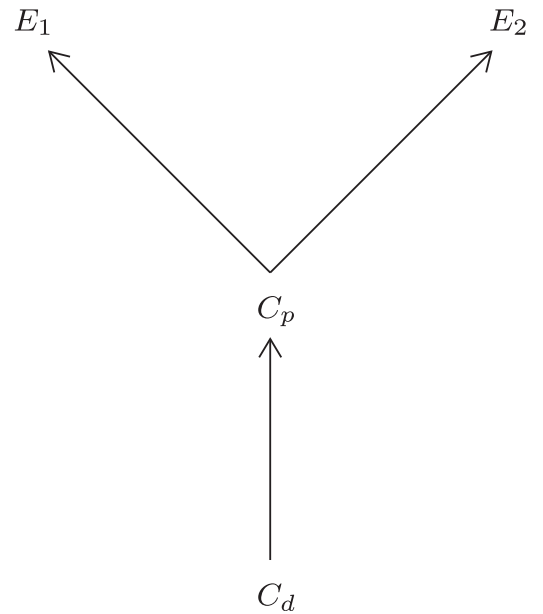

Figure 1. Two questions about screening-off: Does the proximate cause $C_{p}$ screen-off the distal cause $C_{d}$ from $E_{1}$ and from $E_{2}$ ? Does $C_{p}$ screen-off $E_{1}$ from $E_{2}$ ?

odd to say that causation is a relation between variables, but there is really no oddity here. Whether Joe dials Sally's number influences whether Sally's phone rings, and whether it rings influences whether she picks up. This is variable talk. But it also is true that Joe's dialling causes the phone to ring, and the phone's ringing causes Sally to pick up. This is event talk. We stipulate that the occurrence of an event is equivalent to a certain proposition's being true.

As our examples suggest, $X$ screens-off $Y$ from $Z$ means that

$$
\operatorname{Pr}(Y=i \mid X=k)=\operatorname{Pr}(Y=i \mid X=k \& Z=j), \text { for all } i, j, k{ }^{1}
$$

This definition of screening-off applies precisely when all the conditional probabilities are well-defined (meaning that the conditioning propositions all have non-zero probabilities).

With this definition in hand, we can now state what we call the common cause principle $(\mathrm{CC})$ :

If $C$ is the only common cause of two effects $E_{1}$ and $E_{2}$, and neither $E_{i}$ causes the other, then $C$ screens-off $E_{1}$ from $E_{2}$.

What if $E_{1}$ and $E_{2}$ have more than one common cause? Does this mean that CC does not apply? Not at all-the several common causes can be melded together into a single composite cause. This is what we did when we

1 This implies that $\operatorname{Pr}(Y=i \& Z=j \mid X=k)=\operatorname{Pr}(Y=i \mid X=k) \operatorname{Pr}(Z=j \mid X=k)$ for all $i, j, k$; the converse holds also, provided $\operatorname{Pr}(X=k \& Z=j)>0$. 
discussed the parental pair as a single common cause of the two offspring genotypes.

Notice that CC differs from Reichenbach's ([1956]) widely discussed principle of the common cause (RPCC), one version of which reads as follows:

If $E_{1}$ and $E_{2}$ are correlated, ${ }^{2}$ and neither causes the other, they have a screening-off common cause. ${ }^{3}$

CC clearly does not entail RPCC, since RPCC would be false if there were correlated event pairs that are causally unconnected in the sense that neither causes the other and they have no common causes (Sober [2001]; Sober [2008], pp. 230-4) but the existence of such event pairs would not falsify CC. RPCC does not entail $\mathrm{CC}$; the fact that two correlated events have a screening-off common cause does not imply that a complete set of common causes will screen-off.

We turn now to intermediate links. We will say that $C_{p}$ is an intermediate link between $C_{d}$ at $t_{1}$ and an event $E$ at a later time $t_{3}$ precisely when $C_{p}$ occurs between $t_{1}$ and $t_{3}, C_{d}$ causes $C_{p}$, and $C_{p}$ causes $E$. A first attempt at defining an intermediate link principle would be to assert that if $C_{d}$ causes $C_{p}$, and $C_{p}$ causes $E$, and $C_{p}$ is the only intermediate link between $C_{d}$ and $E$, then $C_{p}$ screens-off $C_{d}$ from $E$. However, it is easy to see that this principle will not hold in general; for example, suppose that there is a second, direct pathway by which $C_{d}$ causes $E$ (one that does not pass through $C_{p}$ ), as in the left-hand (curved) link shown in Figure 2. Then $C_{p}$ may fail to screen-off $E$ from $C_{d}$. It may be replied that the thesis of 'no action at a temporal distance (NATD), 4 can be invoked here. The idea is that there must exist a 'representative' event, $R$, on the curved path between $C_{d}$ and $E$, and if we regard the more complete composite cause $\left(C_{p}, R\right)$ as an intermediate link between $C_{d}$ and $E$, then it will now screen-off $E$ from $C_{d}$. Although this recourse to NATD rescues the intermediate link principle we stated, it will not work for certain other cases, such as the two asterisked situations depicted in Figure 2. If an event either causes both $C_{p}$ and $E$ (upper * in Figure 2) or it causes both $C_{d}$ and $E$ (lower * in 2), then not only can $C_{p}$ fail to screen-off $C_{d}$ from $E$ but so too may $\left(C_{p}, R\right)$, for

2 These correlations involve probabilities, not sample frequencies. When $E_{1}$ and $E_{2}$ are dichotomous, correlation means that $\operatorname{Pr}\left(E_{1} \& E_{2}\right) \neq \operatorname{Pr}\left(E_{1}\right) \operatorname{Pr}\left(E_{2}\right)$.

3 Although Reichenbach formulated his principle for dichotomous variables, it is natural to formulate the principle so that it applies to $n$-state variables, for any $n \geq 2$ (Uffink [1999]). Our definitions of screening-off and CC do so.

4 We take NATD to assert:

Along each path from $C$ to $E$ there exists an intermediate link $I$ so that $C$ causes $I$ and $I$ causes $E$.

This principle entails that there are infinitely many intermediate links between $C_{d}$ and $E$. 


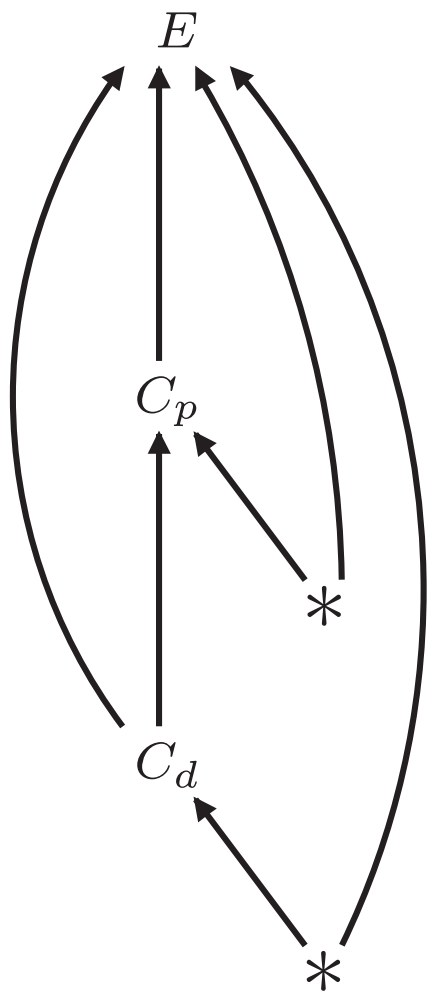

Figure 2. Three cases in which $C_{p}$ can fail to screen-off $C_{d}$ from $E$, involving either a second, direct link from $C_{d}$ to $E$, or events (*) that cause $C_{p}$ and $E$ or that cause $C_{d}$ and $E$. The addition of other causal links, or of other events on existing (or new) causal links, cannot restore the guarantee that $C_{p}$ will screen-off $C_{d}$ from $E$.

any collection $R$ of intermediate links between $C_{d}$ and $E$ (as these must lie on the directed causal path $\left.C_{d} \longrightarrow C_{p} \rightarrow E\right) .{ }^{5,6}$

We can avoid the three counter-examples shown in Figure 2, and set NATD to one side, by formulating the intermediate link principle (IL) as follows:

If $C_{d}$ causes $C_{p}$ which causes $E$, and if every path from $C_{d}$ to $E$, and from any cause of $C_{p}$ to $E$, passes through $C_{p}$, then $C_{p}$ screens-off $E$ from $C_{d}$.

Although we have formulated IL by describing $C_{p}$ as a single variable, there is nothing wrong with thinking of it as a conjunction of variables. For example, if $C_{p}^{1}, C_{p}^{2}, \ldots, C_{p}^{n}$ are the only intermediate links between $C_{d}$ and $E$, (IL) will

5 Or, in addition, on the left-hand curved link if it is also present. Note also that the upper * in Figure 2 could occur before $C_{d}$ though it is shown in Figure 2 as occurring after it.

6 In fact, screening-off must fail in the circumstances described above when the conditions of non-degeneracy and weak influence, which we will discuss in Section 2, are satisfied. 
say that $\left(C_{p}^{1}, C_{p}^{2}, \ldots, C_{p}^{n}\right)$ screens-off $C_{d}$ from $E$ if the assumptions in (IL) are satisfied. The different conjuncts may have different temporal indices, but each must fall between the dates of $C_{d}$ and $E$, namely $t_{1}$ and $t_{3}$. This parallels our earlier point that, when $C C$ talks about $C$ being the 'only' common cause, there is nothing wrong with thinking of it as a conjunction of common causes, and these conjuncts may likewise have different dates.

Neither IL nor CC rules out considering less inclusive sets of intermediate links or common causes; perhaps some of these can be said to screen-off. For example, maybe $C_{p}$ all by itself will screen-off $E_{1}$ from $E_{2}$ in Figure 1, there being no need to mention $C_{d}$. If there are $n$ causal pathways linking $C$ at $t_{1}$ to $E$ at $t_{3}$, maybe $n$ intermediates, one on each pathway, will suffice to screen-off $C$ from $E$.

If the causal relations described in IL and CC are deterministic, then both principles are true, for the simple reason that probabilities of 0 and 1 are 'sticky'. Consider how CC applies to Figure 1. If the causal relations depicted there are deterministic, then $\operatorname{Pr}\left(E_{1}\right.$ at $t_{3} \mid C_{p}$ at $\left.t_{2}\right)$ must equal 0 or 1 , and this entails that $\operatorname{Pr}\left(E_{1}\right.$ at $t_{3} \mid C_{p}$ at $t_{2} \& E_{2}$ at $\left.t_{3}\right)$ must also equal 0 or 1 , so screening-off obtains. On the other hand, if causal relations are not deterministic, the question remains as to why IL and CC should be accepted. We take no stand in what follows on whether IL and CC are true.

CC and IL have different standings in current science. Quantum mechanics has led many to be wary of $\mathrm{CC}$; some even think that quantum mechanical theory and evidence provide good reason to think that CC is false (e.g. Van Fraassen [1982]), though it must be recognized that the DeBroglie-Bohm interpretation of quantum mechanics is deterministic, which means that it entails that $\mathrm{CC}$ is true. So inside quantum mechanics, $\mathrm{CC}$ is controversial. Outside, CC has rarely been doubted; Cartwright ([2002]) is an exception. IL exhibits a different pattern; this principle is widely assumed, both inside of quantum mechanics, and out. When an intermediate link fails to screen-off (and there are no asterisked common causes that might explain why), the usual reaction is that the link must be causally incomplete.

Despite these differences, both IL and CC are consequences of a central principle in Bayes net modelling called the causal Markov condition. To explain why, we begin by defining what it is for a set of variables, $V$, and an acyclic causal graph, $G$, for those variables to satisfy the Markov condition (MC). This is true precisely when, for every $X \in V$,

$$
\operatorname{Pr}(X \mid \operatorname{Par}(X))=\operatorname{Pr}(X \mid \operatorname{Par}(X) \& N D(X)) .
$$

Here $\operatorname{Par}(X)$ are the parents of $X$ (i.e. $X$ 's direct causes in $V$ ), and $N D(X)$ are the non-descendants of $X$ (i.e. variables in $V$ not caused by $X$ ). Thus, MC says that $\operatorname{Par}(X)$ screens-off $X$ from $N D(X)$ for all $X \in V$. 
Let $X, Y, Z$ be any variables or sets of variables. Assuming MC, a sufficient condition for $Z$ to screen-off $X$ from $Y$ is that $X$ and $Y$ are ' $d$-separated' by $Z$ (Theorem 1.2.4 of Pearl ([2000])). Here $d$-separation is a condition that depends just on the structure of the causal graph $G$ within which $X, Y, Z$ sit, and not on the details of the underlying probability distribution that satisfies MC. ${ }^{7}$ If $X$ and $Y$ are not $d$-separated by $Z$, then $Z$ might still screen-off $X$ from $Y$, but this requires precise tuning of the parameters describing the underlying probability distribution (we will provide an example of this in Theorem $5 \mathrm{~b}$ ). ${ }^{8}$

The definition of MC leaves it open when, if at all, a set of variables and a graph will satisfy the Markov condition. We follow Hitchcock's ([2010]) exposition and define the causal Markov condition (CMC) as follows:

If $V$ is a suitable set of variables and $G$ is a true and complete graph of the causal relations among those variables, then $(V, G)$ will obey the Markov condition.

Of course, everything depends on specifying what it means for $V$ to be 'suitable'. To avoid trivialization, this can't be defined to mean that $(V, G)$ exhibits the screening-off properties demanded by MC. Hitchcock ([2010]) points out that the CMC will or may fail if the following conditions aren't satisfied:

(i) $V$ are appropriately distinct.

(ii) $V$ does not include variables that describe distant correlations in quantum systems.

(iii) $V$ are sufficiently fine-grained.

(iv) $V$ doesn't involve mixing populations of different types.

(v) $V$ is causally sufficient; that is, if $V$ contains variables $X$ and $Y$ that have common causes, then those common causes are also in $V$.

(vi) The population to which $(V, G)$ and the probability distribution applies was selected by a procedure that is not biased towards two or more of the variables in the set $V$.

We will understand the CMC to assert that these six conditions suffice for the $\mathrm{MC}$ to be satisfied. We note that the six conditions that go into the CMC are a motley crew. Five of them are 'formal' in the sense that they are neutral about the subject matter of the model under discussion. The sixth is different-it

7 Formally, $Z d$-separates $X$ from $Y$ if and only if each (undirected) path from a variable in $X$ to a variable in $Y$ includes at least one of the following chains, where $i, j$ are any elements of $V$ : (i) $i \rightarrow z \rightarrow j$ or (ii) $i \leftarrow z \rightarrow j$ where $z \in Z$, or (iii) $i \rightarrow m \leftarrow j$ where $m$ is not in $Z$ and no descendant of $m$ is in $Z$ (for further details, see for example Pearl [2009], or Spirtes et al. [1993/2000]).

8 The fact that a cause, $C$, of two effects, $E_{1}$ and $E_{2}$, must screen-off each from the other if $C$ $d$-separates those two effects ensures that our main no-go results (Theorems 4 and 6 and their corollaries) remain true if 'screening-off' is replaced throughout by ' $d$-separates' in their statement. 
excludes causal models that are about quantum mechanical phenomena. We understand why this sixth condition is included but it still gives us pause. If quantum mechanics makes it reasonable to decline to assume the $\mathrm{CMC}$, why should one be so sure that CMC works fine in all other domains? We return to this question at the end of the article.

We now describe how CMC entails both IL and CC:

\section{Theorem 1}

(1a) CMC entails IL: If $C_{d}$ causes $C_{p}$ which causes $E$, and if every path from $C_{d}$ to $E$ and from any cause of $C_{p}$ to $E$, passes through $C_{p}$, then CMC entails that $C_{p}$ screens-off $E$ from $C_{d}$.

(1b) CMC entails CC: If $C$ is the only common cause of two effects $E_{1}$ and $E_{2}$, and neither $E_{i}$ causes the other, then CMC entails that $C$ screens-off $E_{1}$ from $E_{2}$.

To establish Theorem (1a), a case analysis reveals that the only way that $C_{p}$ can fail to $d$-separate $C_{d}$ from $E$ is by one of the situations described in Figure 2 (additional links and events may also be present). However, this is excluded by the antecedent of IL. So, $C_{p} d$-separates $C_{d}$ from $E$ and thereby $C_{p}$ screens-off $C_{d}$ from $E$, by Theorem 1.2.4 of Pearl ([2000]).

To establish Theorem (1b), we simply note that $E_{1}$ and $E_{2}$ are $d$-separated by $C$, and so we may apply Theorem 1.2.4 of Pearl ([2000]), as we did for Theorem (1a). This completes the proof of Theorem 1.

Since IL and CC are both consequences of the CMC, the question arises of why it is worth stating them as separate principles. The reason is that they are logically independent of each other (when CMC is not assumed), as we will show shortly (Corollary 1). But we first observe that even when $\mathrm{CMC}$ is true, there can be causal systems in which there are common causes and intermediate links, where one of these screens-off whereas the other does not. This is the point of our Theorem 2. Returning to Figure 1, we now will take $\left(C_{d}, C_{p}\right)$ to be the ' $C$ ' described in CC and $\left(E_{1}, E_{2}\right)$ to be the ' $E$ ' described in IL.

\section{Theorem 2}

(i) When $C_{d}$ causes $C_{p}$, and $C_{p}$ causes each of $E_{1}$ and $E_{2}$ (with neither $E_{i}$ causing the other) and the $\mathrm{CMC}$ is satisfied, it is possible for $C=\left(C_{d}, C_{p}\right)$ to screen-off $E_{1}$ from $E_{2}$ and yet for $C_{p}$ to fail to screen-off $C_{d}$ from $E_{1}$ or from $E_{2}$. It is also possible for $C_{p}$ to screen-off $C_{d}$ from $E_{1}$ and from $E_{2}$, yet for $C=\left(C_{d}, C_{p}\right)$ to fail to screen-off $E_{1}$ from $E_{2}$.

(ii) Moreover, these two claims also hold when $\left(C_{d}, C_{p}\right)$ is replaced just by $C_{p}$. 
(a)

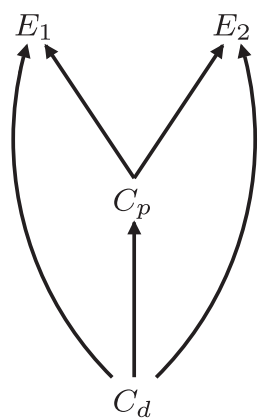

(b)

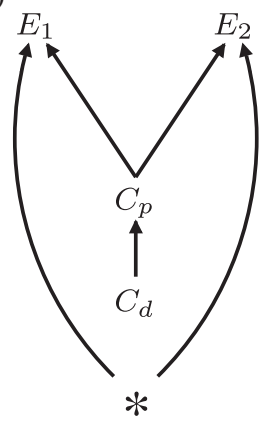

(c)

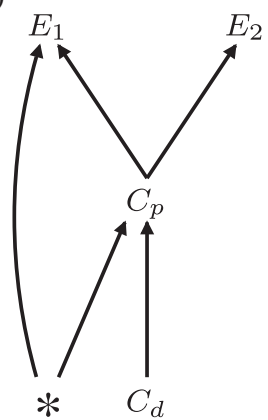

Figure 3. Cases relevant to the proof of Theorem 2.

The proof of the first and second claim in Part (i) of Theorem 2 is illustrated by the two situations shown in Figure $3 \mathrm{a}$ and $\mathrm{b}$, respectively (in part (b), ${ }^{*}$ is a cause of $E_{1}$ and $E_{2}$ that is not a cause of $C_{d}$ ). In both cases it is clear that $C_{d}$ causes $C_{p}$, and $C_{p}$ causes each of $E_{1}$ and $E_{2}$ (with neither $E_{i}$ causing the other). Now, in Figure $3 \mathrm{a}$, although $\left(C_{d}, C_{p}\right) d$-separates $E_{1}$ from $E_{2}, C_{p}$ does not $d$-separate $C_{d}$ from $E_{1}$ or from $E_{2}$ (and so, in particular, there is a probability distribution for which $C_{p}$ does not screen-off $C_{d}$ from $E_{1}$ or from $E_{2}$, by the second part of Theorem 1.2.4 of Pearl ([2000])). Similarly, in Figure 3b, although $C_{p} d$-separates (and so screens-off) $C_{d}$ from $E_{1}$ and $E_{2},\left(C_{d}, C_{p}\right)$ does not $d$-separate $E_{1}$ from $E_{2}$ (and so there is a probability distribution for which $C_{p}$ does not screen-off $E_{1}$ from $E_{2}$ ).

Regarding proof of part (ii), the second claim of part (i) still holds when $\left(C_{d}, C_{p}\right)$ is replaced by $C_{p}$ by repeating the earlier argument with Figure $3 \mathrm{~b}$. However, to establish the first claim of part (i) when $\left(C_{d}, C_{p}\right)$ is replaced by $C_{p}$, requires more care. First observe that, for Figure $3 \mathrm{c}, C_{p} d$-separates $E_{1}$ from $E_{2}$ and $C_{p}$ does not $d$-separate $C_{d}$ from $E_{1}$, and so, as before, we can obtain an example in which $C_{p}$ screens-off $E_{1}$ from $E_{2}$ but fails to screen-off $C_{d}$ from $E_{1}$. However, we wished for something stronger, namely that $C_{p}$ also fails to screen-off $C_{d}$ from $E_{2}$, and this does not hold in Figure 3c. Moreover, adding additional variables and/or directed arcs (for example adding a second event $*$ with arcs to $C_{p}$ and $E_{2}$ ) will not help for the simple reason that if $C_{p} d$-separates $E_{1}$ from $E_{2}$ then $C_{p}$ either $d$-separates $C_{d}$ from $E_{1}$ or $C_{p} d$ separates $C_{d}$ from $E_{2}$.

Thus, to provide an example for the first claim in (ii) we cannot rely just on a $d$-separation argument but must instead resort to an example that requires 'fine balance'. Such examples exist, and we provide an explicit one in the Appendix. This completes the proof of Theorem 2. 
We provide a simple example to illustrate the first situation described in Theorem 2(i), and corresponding to Figure 3a, in which causes are deterministic and trichotomous (and exchangeable). ${ }^{9}$ Suppose that $C_{d}$ and $C_{p}$ are, respectively, the genotype ( $A A, A a$ or $a a$ ) of a female, and of the male who is chosen as a mate by the female. Suppose that $a$ is a recessive allele, and $A$ is dominant, so that individuals of type $A A$ or $A a$ exhibit one phenotype (type- $P$ ), whereas those of type $a a$ exhibit a second (type- $Q$ ); a familiar example from the genetics of eye colour is $P=$ brown and $Q=$ blue. We assume that the population is in Hardy-Weinberg equilibrium, with both alleles present. Suppose further that a type- $Q$ female will only select as her mate a type- $Q$ male, but a type- $P$ female is indifferent to the phenotype of males in her mate selection. Suppose that once the female selects a male they have two offspring. Let $E_{1}$ be the event that the one child is type- $P$ and $E_{2}$ the event that the other child is type- $P$. Regarding the condition that $C_{p}$ does not screen-off $C_{d}$ from $E_{i}$, a sufficient condition for this to hold is that:

$$
\operatorname{Pr}\left(E_{i} \mid C_{p}=a a \& C_{d}=a a\right) \neq \operatorname{Pr}\left(E_{i} \mid C_{p}=a a\right),
$$

and this applies in this example since the left-hand side is zero, whereas the right-hand side is non-zero.

Since CMC entails both IL and CC, the two principles can have opposite truth-values only when CMC is false. For believers in the CMC, it won't make sense to separate CC from IL. But for those who have yet to commit, it is interesting that IL and CC do not necessarily stand or fall together, as the next result asserts.

\section{Corollary 1}

In the setting of Figure 1, probability distributions on $\left(C_{d}, C_{p}, E_{1}, E_{2}\right)$ can be defined so that CC holds and IL fails. Similarly, it is also possible for IL to hold and $\mathrm{CC}$ to fail.

Corollary 1 follows directly from Theorem 2(i); simply take a probability distribution on $\left(C_{d}, C_{p}, E_{1}, E_{2}\right)$ that arises in each of the two scenarios considered in Theorem 2(i) (under CMC) but apply them to the causal setting of Figure 1, where the additional (curved) paths and $*$ events in Figure 3 (used to generate the probability distribution on $\left.\left(C_{d}, C_{p}, E_{1}, E_{2}\right)\right)$ no longer exist. Note that the antecedent of CC and IL both hold in this setting, and in one case the consequent of CC fails, whereas in the other that of IL fails. This completes the proof of Corollary 1.

Although CC and IL are logically independent of each other, there is a property of the screening-off relation that we explore in what follows that

9 Theorem 2 remains true even for dichotomous causes, and with effects that are not deterministically based on the states of their causes. 
pertains to each. Both are formulated in terms of an event that is said to be causally complete. What happens to screening-off when we consider events that are causally incomplete? Telephony and Mendelism show that an incomplete cause may fail to screen-off even though a complete cause succeeds in doing so. Can something stronger be demonstrated - that an incomplete cause must fail to screen-off if a complete cause succeeds? What assumptions about causality are needed to establish this stronger result?

\section{Influence and Non-degeneracy Conditions}

Suppose that $C_{1}$ and $C_{2}$ are each common causes of $E_{1}$ and $E_{2}$, and that $C_{1}$ and $C_{2}$ are not related to each other as cause to effect. An example, discussed earlier, is the two parents who have two offspring. We set to one side the example depicted in Figure 1, in which $C_{d}$ and $C_{p}$ are both common causes of $E_{1}$ and $E_{2}$, and $C_{d}$ causes $C_{p}$. We begin with the case of two common causes and later generalize to the case of $n$.

The variables $C_{1}, C_{2}, E_{1}$, and $E_{2}$ may be dichotomous (in which case we denote the two states as 0 or 1 ) or take values in a larger set of states. ${ }^{10}$ Here we consider the following question: if each possible combination of values for the pair $C_{1}$ and $C_{2}$ (which we denote as $\left(C_{1}, C_{2}\right)$ ) screens-off $E_{1}$ from $E_{2}$, is it possible for one or both of the causes $\left(C_{1}\right.$ or $\left.C_{2}\right)$ individually to screen-off $E_{1}$ from $E_{2}$ ? In what follows, we call $C_{1}$ and $C_{2}$ the constituent (or component) causes and their conjunction, $C_{1} \& C_{2}$, the composite cause. In the genetics example, the genotype of each parent is a constituent cause, whereas the genotype of the parental pair is the composite cause. The theorems we prove in what follows concern the relationship between composite causes screening-off and constituent causes screening-off.

We assume the following two propositions about these constituent causes, which we state now for two or more causes $C_{1}, \ldots, C_{k}$, each of which can assume an arbitrary number of states, and with each cause able to influence one or more effects $E_{1}, \ldots, E_{m}$ :

Non-degeneracy (n-D): For any assignment $s_{1}, \ldots, s_{k}$ of states to $C_{1}, \ldots, C_{k}$, respectively, the events $C_{i}=s_{i}$ have nonzero joint probability; that is

$$
\operatorname{Pr}\left(C_{1}=s_{1} \& C_{2}=s_{2} \& \cdots \& C_{k}=s_{k}\right)>0 .
$$

Weak influence of $C_{i}$ on $E_{j}$ (w-I): For cause $C_{i}$ and effect $E_{j}$, there exists at least one assignment of states for the remaining $k-1$ causes, such that

\footnotetext{
${ }^{10}$ In the case of dichotomous variables $E_{1}$ and $E_{2}$, it is an easy exercise to show that $X$ screens-off $E_{1}$ from $E_{2}$ if conditional independence applies for the events $E_{1}=1$ and $E_{2}=1$.
} 
some change in the state of $C_{i},{ }^{11}$ while holding fixed the value of the remaining $k-1$, changes the probability of at least one state, $s_{j}$, of $E_{j}{ }^{12}$

Notice that condition n-D implies that the causes are less than perfectly correlated, that is, $\operatorname{Pr}\left(C_{i}=s_{i} \mid C_{j}=s_{j}, \forall j \neq i\right)<1$, for each $i$. Regarding w-I, if each cause exerts weak influence on each effect, we say that w-I holds uniformly. Note that, even for two effects, this uniform application of w-I does not require that there exist some change to a state of $C_{i}$ (with all other causes fixed) that simultaneously changes the probability of some state of $E_{1}$ and of some state of $E_{2}$. That stronger requirement is represented by the following proposition:

Strong influence (s-I): For each cause $C_{i}$, there exist at least one assignment of states for the remaining $k-1$ causes, such that some change in the state of $C_{i}$ while holding fixed the value of the remaining $k-1$ changes the probability of at least one state of each of the $m$ effects.

Strong-influence entails weak-influence for the same reason that 'there is a single day that is everyone's birthday' entails 'everyone has a birthday'. The strong influence proposition, s-I will figure in Theorems 4, 5a, and 5b, but it will be dropped and replaced by weak influence, w-I, and by a proposition about causation concerning ordinal invariance in developing Theorems 6 and its corollary. None of our results requires a definition of what causation is.

Because IL and CC describe what must be true when all the intermediate links or all the common causes are in hand, it may seem that neither has any bearing on practical science. Not so. Their applicability is to be found in their contrapositives. Suppose your model says, correctly, that $A$ causes $B$ and $B$ causes $C$, and it then turns out that $B$ does not screen-off $A$ from $C$; IL will conclude that there must be more going on causally than your model acknowledges - there are additional variables and/or causal dependencies you need to take into account. If your model says, correctly, that $C$ is a common cause of $E_{1}$ and $E_{2}$, with neither $E_{i}$ causing the other, and it then turns out that $C$ does not screen-off these two effects from each other, $\mathrm{CC}$ will conclude that your model is incomplete - there are additional common causes out there in nature. So IL and CC underwrite scientific inferences that are both commonplace and sensible. In addition, the causal Markov condition (which entails CC and IL) plays a foundational role in the discovery algorithms (developed by Spirtes et al. ([1993/ 2000]); Verma and Pearl [1990], [1992]; Cooper and Herskovits [1992]) for inferring causal relationships from probabilistic facts; the value of these

11 Here and in what follows, when we speak of 'changing' the state of a cause, we mean interventions in the technical sense that has come to be used in the causal modelling literature. See, for example, Pearl ([2000]), Spirtes et al. ([1993/2000]), and Woodward ([2003]).

12 We will refer to the fixed values of the remaining causes that reveal how a change in $C_{i}$ alters the probability of a state $s_{j}$ of $E_{j}$ as 'revelatory states' for the 'influenced state', $s_{j}$. 
algorithms is not something we dispute. Still, the question remains of whether $\mathrm{CC}$ and IL are true, a topic to which we return at the end of this article.

We end this section by pointing out two restrictions on causes and effects that arise from the previous definitions. First, suppose that $C=\left(C_{1}, \ldots, C_{n}\right)$ is a deterministic cause of $E$ (i.e. the probability that $E$ takes any particular state is either 0 or 1 once we condition on the states of the individual causes in $C$ ), and that $C^{\prime}$ includes $C_{1}, \ldots, C_{n}$ and at least one other event, and that $C^{\prime}$ satisfies n-D. Then $C^{\prime}$ clearly still determines $E$, yet $C^{\prime}$ cannot satisfy w-I by the following result, the proof of which (as with other results which follow) is provided in the Appendix.

\section{Theorem 3a}

If $C$ is a deterministic cause of $E$, and $C^{\prime}$ includes $C$ and at least one other cause, then $C^{\prime}$ must fail to satisfy n-D, or w-I, or both.

We also note that if a common cause, $C$, is to screen-off $E_{1}$ from $E_{2}$, this can place constraints on the number of states that $C$ must possess. More specifically, we have the following result, whose proof is also in the Appendix.

\section{Theorem 3b}

For any two events $E_{1}$ and $E_{2}$, each with $k$ states, if the $k \times k$ matrix $P:=\operatorname{Pr}\left(E_{1}=i \& E_{2}=j\right)$ has non-zero determinant, ${ }^{13}$ then any cause, $C$, with fewer than $k$ states must fail to screen-off $E_{1}$ from $E_{2}$.

In certain settings, $P$ must have a non-zero determinant. One example arises in modelling evolutionary processes, such as DNA site substitutions. Consider any Markov process on $k$ states (for DNA, $k=4$ corresponding to the four nucleotides $\mathrm{A}, \mathrm{C}, \mathrm{G}, \mathrm{T}$ ) that allows transitions between all states at a positive rate, and is in equilibrium. Suppose that a (random) state of the process at time $t_{1}$ evolves from a common ancestor down two independent descendant lineages for a further time $t_{2}$. If $E_{1}$ and $E_{2}$ are the resulting two states (nucleotides) at time $t_{1}+t_{2}$, then $P$ always has strictly positive determinant. However, this does not mean that $P$ has non-zero determinant for all Markovian processes, since some have built-in independence assumptions that insure that the determinant of $P$ is zero. ${ }^{14}$ In these cases Theorem $3 b$ has an extension in

13 A determinant equal to zero is a very strong constraint. Here's a simple visual picture. Suppose each $E_{i}$ has three states; $P$ is therefore a $3 \times 3$ matrix. Consider the three rows (or columns) of $P$ as three points in the three-dimensional cube $(x, y, z)$ where $0<x, y, z<1$. The condition that $\operatorname{det}(P)=0$ is the condition that these three points are colinear.

${ }^{14}$ Consider a Mendelian example. Let $E_{1}$ and $E_{2}$ be the genotypes $(A A, A a, a a)$ of two full sibs, and suppose that the genotype of one parent is Aa whereas that of the second parent is a matter of probability. Each possible state of the genotype of the second parent screens off $E_{1}$ from $E_{2}$. Yet $\operatorname{det}(P)=0$, so Theorem 3 b does not apply. 
which a broader sufficient condition is identified that constrains the number of states a common cause must have if it screens-off. ${ }^{15}$

Theorem $3 \mathrm{~b}$ may be useful in practice. Observing the frequencies of $\left(E_{1}=i\right.$ and $E_{2}=j$ ) for different values of $i$ and $j$ may permit you to estimate the entries in the $P$ matrix, and this may provide sufficiently strong evidence for you to conclude that its determinant is non-zero. If so, you have good reason to conclude that various conjectured common causes cannot screen-off.

\section{A No-Go Theorem for Two Dichotomous Causes and its Limitations}

For dichotomous effects and just two common causes, at least one of which is dichotomous, n-D and s-I suffice to establish a conflict between a composite cause's screening-off and a constituent cause's doing so. Although we will prove no-go theorems that cover larger numbers of variables (both dichotomous and non-dichotomous), these will require more intricate conditions. For starters, we have:

\section{Theorem 4 (dichotomous cause(s) and effects)}

Suppose that $C_{1}$ and $C_{2}$ are common causes of the dichotomous effects $E_{1}$ and $E_{2}$, that n-D and s-I are satisfied, and that $C_{2}$ is dichotomous. Then the composite cause, $\left(C_{1}, C_{2}\right)$, screens-off $E_{1}$ from $E_{2}$ only if $C_{1}$ fails to do so. Moreover, if both causes are dichotomous and the composite cause screens-off $E_{1}$ from $E_{2}$, then neither component cause screens-off. ${ }^{16}$

Theorem 4 also applies to the case of intermediate links: Suppose that $C$ causes $I_{1}$ and $I_{2}$, and each of these causes $E$, where all these variables are dichotomous. If n-D and s-I are satisfied (with $C_{1}=I_{1}, C_{2}=I_{2}, E_{1}=E$, $\left.E_{2}=C\right),{ }^{17}$ then if the composite $\left(I_{1}, I_{2}\right)$ screens-off $C$ from $E$, each of the constituents $I_{1}$ and $I_{2}$ fails to do so.

Notice that Theorem 4 requires at least one of the causes to be dichotomous. What if none of them is? It turns out that extending Theorem 4 to nondichotomous causes necessarily requires imposing additional conditions, as the following result makes clear.

15 Regardless of the value of the $\operatorname{det}(P)$, if $P$ has rank equal to $r$, then any common cause with fewer than $r$ states fails to screen-off $E_{1}$ from $E_{2}$ (see the proof of Theorem $3 \mathrm{~b}$ ). The 'rank' of a matrix is the number of its linearly independent rows (or, equivalently, columns). $E_{1}$ and $E_{2}$ are independent precisely when $P$ has rank 1 . At the other extreme, a $k \times k$ matrix has maximal rank $k$ iff its determinant is non-zero.

16 This theorem strengthens a result derived by Sober ([1988], pp. 98-9).

17 Here and elsewhere, we allow a 'cause' to also be an 'effect' (for example the event $C_{p}$ in Figure 1 ); formally, there is no problem in doing this as the properties we describe relate to events generally. 
Table 1. $\operatorname{Pr}\left(E_{1}=1 \mid C_{1}=g \& C_{2}=h\right)$

\begin{tabular}{lccc}
\hline & $C_{2}=1$ & $C_{2}=2$ & $C_{2}=3$ \\
$C_{1}=1$ & 0.2 & 0.3 & 0.4 \\
$C_{1}=2$ & 0.3 & 0.4 & 0.5 \\
$C_{1}=3$ & 0.4 & 0.5 & 0.6 \\
\hline
\end{tabular}

Table 2. $\operatorname{Pr}\left(E_{2}=1 \mid C_{1}=g \& C_{2}=h\right)$

\begin{tabular}{lccc}
\hline & $C_{2}=1$ & $C_{2}=2$ & $C_{2}=3$ \\
$C_{1}=1$ & 0.2 & 0.4 & 0.3 \\
$C_{1}=2$ & 0.4 & 0.6 & 0.5 \\
$C_{1}=3$ & 0.3 & 0.5 & 0.4 \\
\hline
\end{tabular}

\section{Theorem 5a (both causes non-dichotomous)}

There exist two non-dichotomous causes $C_{1}$ and $C_{2}$ satisfying n-D and s-I for two effects $E_{1}$ and $E_{2}$, where each of $\left(C_{1}, C_{2}\right), C_{1}$ and $C_{2}$ screens-off $E_{1}$ from $E_{2}$. Moreover, we can require that the two causes each have three equiprobable states, and are independent of each other.

We now describe an example to justify Theorem 5a. Suppose we have two causes $C_{1}$ and $C_{2}$, each of which has three states, along with two dichotomous effects, $E_{1}$ and $E_{2}$, which are screened-off from each other by the pair $\left(C_{1}, C_{2}\right)$. Now, suppose that the conditional probabilities of the events $E_{1}=1$ and $E_{2}=$ 1 , given the possible combination of states of the causes are as shown in Tables 1 and 2, respectively. Notice that, as we increase the numerical state of $C_{1}$ (for each fixed state of $C_{2}$ ), the conditional probability that $E_{1}=1$ increases linearly (Table 1 ) whereas the conditional probability that $E_{2}=1$ increases and then decreases (Table 2).

As a simple example, let $C_{1}$ be the number of rest days $(1,2$ or 3$)$ that a runner takes every four days while training for a marathon, $E_{1}$ be the event that she has no injuries or pain at the start of the race (where increasing rest helps), and $E_{2}$ the event that she wins (where some rest, but not too much, is best). Tables 1 and 2 also show a similar pair of contrasting behaviours if we fix a value of $C_{1}$ and vary $C_{2}$ (in the marathon setting, $C_{2}$ might measure the degree of supportiveness of the running shoes used during training - as with $C_{1}$, larger $C_{2}$ values increase the probability of $E_{1}$, but there is again likely to be a sweet spot for the event $E_{2}$ ).

For the values in Tables 1 and 2, it can be shown that if the causes are independent, and for $i=1,2$ we set

$$
\operatorname{Pr}\left(C_{i}=1\right)=\frac{1}{9} \text { and } \operatorname{Pr}\left(C_{i}=2\right)=\operatorname{Pr}\left(C_{i}=3\right)=\frac{4}{9},
$$


then $C_{1}$ screens-off $E_{1}$ from $E_{2}$, thereby justifying Theorem 5a (a more formal proof is provided in the Appendix). Moreover, by the symmetry in the tables, $C_{2}$ also screens-off $E_{1}$ from $E_{2}$. We note that the particular solution we have described is not unique, but any solution requires certain equations to be satisfied exactly.

Theorem 5a addressed the question of whether Theorem 4, which concerns two dichotomous causes, breaks down when we depart from the setting of dichotomous causes. The example described is germane because it involves causes that have three states. Now we consider another departure from the narrow compass of Theorem 4-we show that this theorem also breaks down when there are more than two dichotomous causes. Again, the increase in number that needs to be considered is modest-we move from two to three. For three dichotomous causes, it is possible for the composite cause to screen-off and for each of the three constituent causes to screen-off as well.

\section{Theorem $\mathbf{5 b}$}

There exist three independent and dichotomous causes $C_{1}, C_{2}, C_{3}$ and two dichotomous effects $E_{1}$ and $E_{2}$ that satisfy n-D and s-I, for which: (i) $\left(C_{1}, C_{2}\right.$, $C_{3}$ ) screens-off $E_{1}$ from $E_{2}$; (ii) $C_{i}$ screens-off $E_{1}$ from $E_{2}$ for each $i=1,2,3$; (iii) $\left(C_{1}, C_{2}\right),\left(C_{2}, C_{3}\right)$, and $\left(C_{1}, C_{3}\right)$ each fail to screen-off $E_{1}$ from $E_{2}$.

To establish this result we describe a bar game that involves three players, each of whom reveals either a clenched fist or an open hand; they do so simultaneously (by the usual 'three shakes method'). A person wins this game if he or she reveals a hand that differs from what the other two players present, in which case the latter two lose. Otherwise (i.e., if all three reveal a clenched fist or all reveal an open hand) the game is a draw. Suppose each player decides his or her strategy independently and chooses between the two strategies with equal probability. If there is a winner, this player must drink a glass of tequila (with probability 1), whereas each of the two losers is required to toss a fair coin, and drink a glass of tequila if and only if the toss lands heads. If the game ends in a draw, then one of the three players is selected uniformly at random (for example, by rolling a fair die with two faces assigned to each person) and that person drinks a glass of tequila.

Let $E_{1}$ be the event that player 1 drinks a glass of tequila and $E_{2}$ the event that player 2 drinks a glass of tequila. We won't model whether player 3 partakes. Let $C_{i}$ be the hand behaviour of player $i$ (for $i=1,2,3$; with $0=$ open hand, $1=$ clenched fist). It is clear that $n-D$ applies since the three causes are independent and $\operatorname{Pr}\left(C_{i}=1\right)=1 / 2$. Moreover, the strong influence condition, s-I, applies, since if any two players select the same strategy (say 'clenched fist'), then changing the strategy for the remaining player from that same strategy to the alternative alters the probability of the 
event $E_{1}=1$ and of the event $E_{2}=1$ (because we thereby change from a draw to a situation in which there is a winner and two losers). Property (i) is clear by the independence of the drinking behaviours after the hands are held forth. For property (ii), the strong symmetry in the game entails that the conditional probability $\operatorname{Pr}\left(E_{j}=1 \mid C_{i}=x\right)$ takes the same values for both $j$, all three values of $i$, and both values of $x$, and this probability equals

$$
\operatorname{Pr}\left(E_{j}=1 \mid C_{i}=x\right)=\frac{1}{4} \cdot 1+\frac{1}{2} \cdot \frac{1}{2}+\frac{1}{4} \cdot \frac{1}{3}=\frac{7}{12},
$$

by considering the probability of win, loss, or draw, respectively, for player $j$. Similarly,

$$
\operatorname{Pr}\left(E_{1}=1 \& E_{2}=1 \mid C_{i}=x\right) \text { is also independent of } i \text { and } x \text { and }
$$

$$
\operatorname{Pr}\left(E_{1}=1 \& E_{2}=1 \mid C_{i}=x\right)=\frac{1}{4} \cdot 1 \cdot \frac{1}{2}+\frac{1}{4} \cdot \frac{1}{2} \cdot 1+\frac{1}{4} \cdot\left(\frac{1}{3}\right)^{2}+\frac{1}{4} \cdot\left(\frac{1}{2}\right)^{2}=\frac{49}{144},
$$

by considering the probability that player 1 wins, player 2 wins, both players draw, and player 3 wins, respectively. It is now apparent that this quantity is the square of $\operatorname{Pr}\left(E_{j}=1 \mid C_{i}=x\right)$, which establishes property (ii). Property (iii) follows from the following corollary to Theorem 4 (also proved in the Appendix):

\section{Corollary 2}

Suppose there are $k>1$ dichotomous causes $\left(C_{1}, \ldots, C_{k}\right)$ which jointly screen-off $E_{1}$ from $E_{2}$ (both dichotomous) and satisfy n-D and s-I. Then $\left(C_{1}, \ldots, C_{k-1}\right)$ cannot screen-off $E_{1}$ from $E_{2}$. The same conclusion applies for any other conjunction of $k-1$ causes.

This completes the proof of Theorem $5 b .^{18}$

The results described in this section have two sorts of significance for philosophical discussions of screening-off and of common cause explanations. The first arises from the fact that Reichenbach's ([1956]) discussion of the principle of the common cause focused on the case of dichotomous events and a fair amount of subsequent philosophical discussion has followed his lead, perhaps tacitly assuming that the transition from two states to $n$ would not make any interesting difference. Theorems $4,5 \mathrm{a}$, and $5 \mathrm{~b}$ show that the move from two states to three sometimes involves a dramatic change. The second lesson pertains to the fact that philosophical discussions of screening-off often focus on cases in which screening-off is robust; the qualitative 'causal structure' of a system suffices to establish that various screening-off relations obtain in the sense that screening-off does not depend on the values that various constituent

18 Theorems $5 \mathrm{a}$ and $5 \mathrm{~b}$ involve examples that are a bit complicated. We searched for simpler examples, but failed to find any. 
probabilities happen to have. This is true of the two examples with which we began. Before caller-ID, the ringing of Sally's telephone screened-off Joe's dialling her number from her picking up, and this relation held independently of the probability that Joe dials her number and independently of the probability that Sally picks up her phone conditional on its ringing. In Mendelian reproduction, the parental genotype screens-off one offspring's genotype from the other's, and this is independent of how probable it is that the parental genotype occurs in the first place. Even if you add a non-zero mutation rate so that the probability of an offspring's genotype, conditional on the genotypes of its parents, takes values that differ from the usual Mendelian assignments, screening-off still holds true. But screening-off isn't always robust; in the examples described in this section, screening-off is fragile, depending as it does on the exact value of this or that probability. ${ }^{19}$

We end this section by noting that the strong influence condition, s-I, requires that some particular change in the state of $C_{i}$ (with other causes suitably fixed) must change the probability of certain states of both $E_{1}$ and of $E_{2}$. It is a consequence of s-I that $\left(C_{1}, C_{2}\right)$ can satisfy s-I for $E_{1}$, and also satisfy s-I for $E_{2}$, and yet fail to satisfy s-I for the pair $\left(E_{1}, E_{2}\right)$ (we give an example shortly).

This raises a question: does Theorem 4 still hold if we replace the assumption of strong influence with the assumption of weak influence? That is, does the theorem go through if we assume only that each cause $C_{i}$ influences the probability of a state of $E_{1}$ under one change in state in $C_{i}$ (with other causes suitably fixed) and that $C_{i}$ influences the probability of a state of $E_{2}$ by a (possibly different) change in state of $C_{i}$ (with other causes suitably re-fixed)? This condition, weaker than s-I, is what we referred to as weak influence w-I holding uniformly, and it is easily seen that Theorem 4 can fail under it. For example, suppose that $C_{1}$ and $C_{2}$ are independent fair tosses of a coin, and that $E_{1}$ is a dichotomous event that occurs with probability $p$ when both tosses are heads and with probability $q \neq p$ otherwise. Let $E_{2}$ denote a second dichotomous event that occurs with probability $p$ when both tosses are tails and with probability $q \neq p$ otherwise. Define the joint probability distributions for $\left(E_{1}\right.$, $\left.E_{2}\right)$ conditional on values for $\left(C_{1}, C_{2}\right)$ so that $\left(C_{1}, C_{2}\right)$ screens-off $E_{1}$ from $E_{2}$. Then the weak influence condition, w-I, applies uniformly, and n-D also holds, yet it is easily checked that all three of $\left(C_{1}, C_{2}\right), C_{1}$, and $C_{2}$ screen-off

19 Fragile screening-off involves a violation of what Spirtes et al. ([1993], [2000]) call 'faithfulness' and Pearl ([2009]) calls 'stability'. Sprites et al. use the assumption of faithfulness to derive a number of theorems. Cartwright ([2007], Chapter 6) and Hitchcock ([2010]) describe plausible examples to argue that faithfulness is not a necessary condition for causal relations in nature. Zhang and Spirtes ([2008]) discuss conditions under which faithfulness fails and how its failure can be detected. Pearl and Hitchcock suggest an epistemic reason for wanting models to be faithful - these models are more parsimonious (in that they deploy fewer adjustable parameters) than models that permit or require faithfulness to be violated. A further form of fragile screening-off is the notion of 'context-specific independence' (Boutilier et al. ([1996]). 
$E_{1}$ from $E_{2}$ (and so, by Theorem 4, s-I does not hold, as can easily be seen directly).

\section{A More General No-Go Theorem: Allowing Several Causes, Possibly Non-dichotomous}

In light of Theorems $5 \mathrm{a}$ and $5 \mathrm{~b}$, we turn to a new topic. If the composite cause $\left(C_{1}, C_{2}\right)$ screens-off $E_{1}$ from $E_{2}$, what new constraint can be introduced that has consequences for whether the constituent causes also screen-off the effects from each other, when all these events can take more than two possible values? The new constraint we will explore is that of ordinal invariance.

Accordingly, we regard $C_{1}$ and $C_{2}$ as random variables taking values in finite sets $G$ and $H$, respectively, and $E_{1}$ and $E_{2}$ as random variables taking values in sets $J_{1}$ and $J_{2}$, respectively. Let $C_{g h}$ and $E_{i j}$ denote the conjunctive events $C_{1}=g \& C_{2}=h$ and $E_{1}=i \& E_{2}=j$, respectively, for all possible combinations of states ( $i, j$ for $E_{1}, E_{2}$ and $g, h$ for $C_{1}, C_{2}$, respectively). For a state $g$ in $G$ and $j_{1}$ in $J_{1}$ and $j_{2}$ in $J_{2}$ we say that $E_{1}$ and $E_{2}$ satisfy ordinal invariance relative to the ordered quintuple $\left(C_{1}, C_{2}, g, j_{1}, j_{2}\right)$ if one of the following holds:

For all states $h$ and $h^{\prime}$ of $C_{2}$,

$$
\begin{aligned}
& \operatorname{Pr}\left(E_{1}=j_{1} \mid C_{g h}\right)>\operatorname{Pr}\left(E_{1}=j_{1} \mid C_{g h^{\prime}}\right) \Rightarrow \operatorname{Pr}\left(E_{2}=j_{2} \mid C_{g h}\right)>\operatorname{Pr}\left(E_{2}=j_{2} \mid C_{g h^{\prime}}\right) \\
& \text { or, for all states } h \text { and } h^{\prime} \text { of } C_{2}, \\
& \operatorname{Pr}\left(E_{1}=j_{1} \mid C_{g h}\right)>\operatorname{Pr}\left(E_{1}=j_{1} \mid C_{g h^{\prime}}\right) \Rightarrow \operatorname{Pr}\left(E_{2}=j_{2} \mid C_{g h}\right)<\operatorname{Pr}\left(E_{2}=j_{2} \mid C_{g h^{\prime}}\right) .
\end{aligned}
$$

In other words, changing the value of $C_{2}$ while holding fixed the value of $C_{1}$ at $g$ always has the same directional effect on the probability of a state of $E_{2}$ that it has on the probability of some state of $E_{1}$, or always has the opposite directional effect.

We pause to describe a natural setting in which ordinal invariance always applies when $J_{1}=J_{2}=J$. We say that $E_{1}$ and $E_{2}$ are conditionally exchangeable (relative to $C_{1}, C_{2}$ ) precisely when

$$
\operatorname{Pr}\left(E_{i j} \mid C_{g h}\right)=\operatorname{Pr}\left(E_{j i} \mid C_{g h}\right)
$$

holds for all values of $i, j \in J$ and $g \in G, h \in H$. For example, this exchangeability condition holds if $E_{1}$ and $E_{2}$ are the genotypes of two children born to the same two parents where the parents have genotypes $g$ and $h$.

\section{Lemma 1}

If $E_{1}, E_{2}$ are conditionally exchangeable relative to $C_{1}, C_{2}$, then $E_{1}$ and $E_{2}$ satisfy ordinal invariance relative to $\left(C_{1}, C_{2}, g, j, j\right)$ for all $g$ in $G$ and $j \in J$. 


\section{Proof}

$$
\begin{aligned}
& \operatorname{Pr}\left(E_{1}=j \mid C_{g h}\right)=\sum_{j^{\prime} \in J} \operatorname{Pr}\left(E_{1}=j \& E_{2}=j^{\prime} \mid C_{g h}\right) \\
& =\sum_{j^{\prime} \in J} \operatorname{Pr}\left(E_{1}=j^{\prime} \& E_{2}=j \mid C_{g h}\right)=\operatorname{Pr}\left(E_{2}=j \mid C_{g h}\right),
\end{aligned}
$$

from which Lemma 1 follows.

We now use the concept of ordinal invariance to state our next result:

Theorem 6 (No restriction to dichotomous events, and the influence condition is weakened):

Suppose that $C_{1}$ and $C_{2}$ satisfy n-D and are common causes of effects $E_{1}$ and $E_{2}$, which take states in sets $J_{1}$ and $J_{2}$, respectively. Suppose that $C_{2}$ weakly influences $E_{1}$, and for some associated revelatory state $g$ of $C_{1}$ for an influenced state $j$ of $E_{1},{ }^{20}$ the effects $E_{1}$ and $E_{2}$ satisfy ordinal invariance relative to $\left(C_{1}, C_{2}, g, j, j^{\prime}\right)$. Then, if the composite $\left(C_{1}, C_{2}\right)$ screens-off $E_{1}$ from $E_{2}, C_{1}$ by itself fails to do so. ${ }^{21}$

The ordinal invariance of $E_{1}$ and $E_{2}$ (relative to the ordered quintuple) means that changing $C_{2}$ while holding the state of $C_{1}$ fixed at $g$ must have the same (or the opposite) ordinal effect on $E_{2}$ that it has on $E_{1}$; this definition says nothing about how changing $C_{1}$ while holding fixed $C_{2}$ must impact on both $E_{1}$ and $E_{2}$. These asymmetries (in requiring w-I to apply only for $C_{2}$ and in the concept of ordinal invariance as so far defined) are reflected in the conclusion drawn by Theorem 6 , which is about $C_{1}$ 's failing to screen-off. In Corollary 3 , we describe the consequence of having the weak influence condition satisfied by each cause and having ordinal invariance pertain to both sorts of manipulations of $C_{1}$ and $C_{2}$; in addition, this new theorem generalizes to the case of $n \geq 2$ causes.

If w-I applies symmetrically to $n \geq 2$ causes, the conclusion of Theorem 6 can be strengthened to the statement that none of the individual causes screen-off $E_{1}$ from $E_{2}$. But first, some notation: suppose that $C_{1}, C_{2}, \ldots, C_{n}$ are each common causes of the effects $E_{1}$ and $E_{2}$. For a state $g_{i}$ of $C_{i}$, and an assignment, $H$, of states to the remaining $n$ - 1 causes, we will use the shorthand $C_{g_{i} H}$ to denote the event that $C_{i}$ is in state $g_{i}$, and the remaining causes are in states specified by $H$ (this generalizes our earlier notation from two to $n$ causes).

\footnotetext{
${ }^{20}$ Recall (footnote 12): a revelatory state of $C_{1}$ for influenced state $j$ of $E_{1}$ is a state of $C_{1}$ under which some change to $C_{2}$ alters the probability of the state $j$ for $E_{1}$.

21 In light of Lemma 1, Theorem 6 applies also if ordinal invariance is replaced by exchangeability.
} 


\section{Corollary 3}

Suppose that $C_{1}, C_{2}, \ldots, C_{n}$ are common causes of effects $E_{1}$ and $E_{2}$, which take states in sets $J_{1}$ and $J_{2}$, respectively (where these two sets may differ in size), and that n-D applies. Suppose further that, for each $C_{i}$, there exists a state $g_{i}$, and there exist states $j_{i}$ for $E_{1}$ and $j_{i}^{\prime}$ for $E_{2}$ to which the following two conditions apply:

(w-I $\mid \mathrm{C}_{i}$ ) (Influence on $E_{1}$ when a state of $C_{i}$ is held fixed): A change can be made to one of the causes (different from $C_{i}$ ) while holding all of the other causes fixed (with $C_{i}$ set to $g_{i}$ ) that changes the probability of the event $E_{1}=j_{i}{ }^{22}$

$\left(\mathrm{OI} \mid \mathrm{C}_{i}\right)$ (Ordinal Invariance when a state of $C_{i}$ is held fixed) If $C_{i}$ is in state $g_{i}$, then every change to the states $H$ of the other causes that increases $\operatorname{Pr}\left(E_{1}=j_{i} \mid \mathrm{C}_{g_{i} H}\right)$ must always increase $\operatorname{Pr}\left(E_{2}=j_{i}^{\prime} \mid C_{g_{i} H}\right)$ or every such change must always decrease it. ${ }^{23}$

Then $\left(C_{1}, C_{2}, \ldots, C_{n}\right)$ screens-off $E_{1}$ from $E_{2}$ only if none of the $n$ component causes screen-off.

As with Theorem 4, Theorems 6 and Corollary 3 also apply to the case of intermediate links: Suppose that $C$ causes $I_{1}$ and $I_{2}$, and each of these causes $E$. Suppose that n-D is satisfied by $I_{1}$ and $I_{2}$, that the influence condition on $E_{1}$ for fixed states of $I_{1}$ and of $I_{2}$ applies in the statement of Corollary 3 (taking $E_{1}=E$ and $E_{2}=C$ ), and that ordinal invariance holds in this setting. Then, if the composite $\left(I_{1}, I_{2}\right)$ screens-off $C$ from $E$, each of the constituents $I_{1}$ and $I_{2}$ fails to do so.

Notice that the first and second parts of Theorem 4 are corollaries of Theorem 6 and Corollary 3, respectively, since s-I implies $\left(\mathrm{OI} \mid \mathrm{C}_{i}\right)$ when $C_{2}$ is dichotomous. Also note that Theorem 6 and its corollary hold, whether the states of the composite cause determine the state of each $E_{i}$ or merely confer nonextreme probabilities on them.

Theorem 6 and Corollary 3 describe a fairly generic type of situation in which it is impossible for a composite cause and its constituent causes to all screen-off one event from another. This clash between composite and constituent screening-off is not inevitable; we had to assume non-degeneracy, the

22 Condition (w-I $\left.\mid \mathrm{C}_{i}\right)$ implies the weaker condition that $\operatorname{Pr}\left(E_{1}=j_{i} \mid \mathrm{C}_{g_{i} H}\right)$ does not always take the same value as $H$ varies, and this is all we require in the proof.

23 Formally, we require that either

(a) for all $H$ and $H^{\prime}$

$$
\operatorname{Pr}\left(E_{1}=j_{i} \mid C_{g_{i} H}\right)>\operatorname{Pr}\left(E_{1}=j_{i} \mid C_{g_{i} H^{\prime}}\right) \Rightarrow \operatorname{Pr}\left(E_{2}=j_{i}^{\prime} \mid C_{g_{i} H}\right)>\operatorname{Pr}\left(E_{2}=j_{i}^{\prime} \mid C_{g_{i} H^{\prime}}\right),
$$

or (b) for all $H$ and $H^{\prime}$

$$
\operatorname{Pr}\left(E_{1}=j_{i} \mid C_{g_{i} H}\right)>\operatorname{Pr}\left(E_{1}=j_{i} \mid C_{g_{i} H^{\prime}}\right) \Rightarrow \operatorname{Pr}\left(E_{2}=j_{i}^{\prime} \mid C_{g_{i} H}\right)<\operatorname{Pr}\left(E_{2}=j_{i}^{\prime} \mid C_{g_{i} H^{\prime}}\right) .
$$


weak influence condition w-I and ordinal invariance to show that it exists. The non-degeneracy condition is very widely satisfied; causes very often are less than perfectly correlated and have probabilities that are strictly between 0 and 1. The weak influence condition w-I strikes us as a definition; if $C$ is a direct cause of $E$, then some change in the state of $C$ must in some setting make some difference to the probability of some state of $E .^{24}$ The ordinal invariance condition is widely satisfied, but it is not a definitional truth. It is the real source of the conflict between composite and constituent causes screening-off. Deny this assumption (as we did in the example used to illustrate Theorem 5a) and it is possible for harmony to replace conflict. But possibility is not necessity; we saw in that example that composite and constituent causes all screen-off only when various probabilities are set just so (and so faithfulness is violated). In the next section, we try to make it easier to understand the concept of ordinal invariance.

\section{Examples Illustrating Corollary 3 and Theorem 5a}

To make Corollary 3 easier to grasp, we describe an example that illustrates what it says when there are just two common causes. Table 3 provides conditional probability values for one of the states of one of the effects (viz. $E_{1}=1$ ); Table 4 provides values for the probability of a state of the other effect (viz. $E_{2}$ $=2$ ). In this example, each cause has three states.

The weak influence condition described in Corollary 3 concerns Table 3 taken by itself. It requires simply that there be a row in which the entries are not all the same, and that the same be true for a column. Several rows and columns in Table 3 satisfy this condition, but we will consider $C_{1}=3$ and $C_{2}=$ 2 (which are in bold). We focus on this row and column because of what the ordinal invariance condition, $\left(\mathrm{OI} \mid \mathrm{C}_{i}\right)$, says; this condition concerns the relationship of Table 3 to Table 4.

A sufficient condition for $\left(\mathrm{OI} \mid \mathrm{C}_{i}\right)$ to hold is that there is a row in Table 3 in which not all entries are the same and a column that has the same property where the entries in that row and column are mirrored (or countermirrored) by the corresponding row and column in Table $4 .{ }^{25}$ Mirroring means that all inequalities in a row and column in Table 3 are preserved in its counterpart row and column in Table 4; equalities need not be preserved (and they are not for the column $C_{2}=2$ ). Countermirroring means that all inequalities are reversed. The bold row $C_{1}=3$ and column $C_{2}=2$ in Table 4 mirror those in Table 3 .

24 As noted earlier, the weak influence condition w-I used in Corollary 3 is weaker than the strong influence condition s-I used in earlier theorems. However, strong influence s-I is entailed by the conjunction of n-D, w-I, and $(\mathrm{OI} \mid \mathrm{C})$. Corollary 3 has a stronger antecedent and a stronger consequent than Theorem 4 does.

25 This condition is not necessary, since Corollary 3 allows for $j_{1} \neq j_{2}$ and $j_{1}^{\prime} \neq j_{2}^{\prime}$. 
Table 3. $\operatorname{Pr}\left(E_{1}=j \mid C_{1}=g \& C_{2}=h\right)$ where suppose $j=1$

\begin{tabular}{lccc}
\hline & $C_{2}=1$ & $C_{2}=2$ & $C_{2}=3$ \\
$C_{1}=1$ & 0.1 & $\mathbf{0 . 1}$ & 0.1 \\
$C_{1}=2$ & 0.2 & $\mathbf{0 . 1}$ & 0.5 \\
$C_{1}=3$ & $\mathbf{0 . 1}$ & $\mathbf{0 . 3}$ & $\mathbf{0 . 2}$ \\
\hline
\end{tabular}

Table 4. $\operatorname{Pr}\left(E_{2}=j^{\prime} \mid C_{1}=g \& C_{2}=h\right)$ where suppose $j^{\prime}=2$

\begin{tabular}{cccc}
\hline & $C_{2}=1$ & $C_{2}=2$ & $C_{2}=3$ \\
$C_{1}=1$ & 0.3 & $\mathbf{0 . 2}$ & 0.2 \\
$C_{1}=2$ & 0.1 & $\mathbf{0 . 3}$ & 0.2 \\
$C_{1}=3$ & $\mathbf{0 . 2}$ & $\mathbf{0 . 7}$ & $\mathbf{0 . 4}$ \\
\hline
\end{tabular}

In summary: In the statement of Corollary 3 , if we take $g_{1}=3$ and $g_{2}=2$ (and take $j_{1}=j_{2}=j=1$ and $\left.j_{1}^{\prime}=j_{2}^{\prime}=j^{\prime}=2\right)$, then the two conditions ( $\left.\mathrm{w}-\mathrm{I} \mid \mathrm{C}_{i}\right)$ and $\left(\mathrm{OI} \mid \mathrm{C}_{i}\right)$ are simultaneously satisfied for each of the two causes $(i=1,2)$. This is true even though the column $C_{2}=3$ in Table 4 neither mirrors nor countermirrors its counterpart in Table 3. Notice also that ordinal invariance means that there are inequalities in the table for a state of $E_{1}$ that are mirrored or countermirrored in the table for a state of $E_{2}$; the condition does not say that there are inequalities in the table for a state of $E_{2}$ that are mirrored or countermirrored in the table for a state of $E_{1}$. So our notion of ordinal invariance is weaker than one that requires full symmetry between the two effects.

Theorem 6 and Corollary 3 place no restrictions on how many states $E_{1}$ and $E_{2}$ have (nor do these results require that $E_{1}$ and $E_{2}$ have the same number of states). If there are more than two effects, Theorem 6 and its corollary apply directly since the screening-off of three or more effects from each other by causes means just that any two effects are screened-off from each other by those same causes. Theorem 6 and its corollary also impose no requirement that $C_{1}$ and $C_{2}$ have the same number of states, though this happens to be true in the above example.

Given Corollary 3 (which describes a situation in which the composite cause screens-off only if no constituent cause screens-off), how can Theorem 5a (which describes a situation in which composite and constituent causes all screen-off) be true? The answer is that Theorem $5 \mathrm{a}$ is true because there are cases in which ordinal invariance is violated, whereas Corollary 3 describes a consequence of ordinal invariance. Ordinal invariance is violated in the example used to illustrate Theorem 5a; it is clear from Tables 1 and 2 that there is no mirroring or counter-mirroring. 
Our last no-go results (Theorem 6 and Corollary 3), and some of the theorems that preceed them, are fruitfully compared with an elegant no-go theorem derived by Hofer-Szabó and Rédei ([2004]). Their result concerns what they call a 'Reichenbachian common cause system' (RCCS) for two events $\left(E_{1}, E_{2}\right)$. Such a system involves a common cause, $C$, (whether individual or composite) and two of its effects, $E_{1}$ and $E_{2}$, such that (i) $C$ screens-off $E_{1}$ from $E_{2}$ and (ii) if the state of $C$ is changed in any way, this alters the probabilities of $E_{1}$ and of $E_{2}$ (and the alteration increases the probability of $E_{1}$ precisely when it also increases the probability of $E_{2}$ ). Proposition 4 of Hofer-Szabó and Rédei ([2004]) states that if $C$ is an RCCS for $\left(E_{1}, E_{2}\right)$, then any cause $C^{\prime}$ that is coarser or finer than $C$ must fail to form an RCCS for $\left(E_{1}, E_{2}\right)$. Here a cause $C^{\prime}$ is 'coarser' than $C$ precisely if the states of $C^{\prime}$ correspond to disjoint subsets of the states of $C$, and the event that $C^{\prime}=s$ is the event that $C \in s$. For example, if $C$ is the composite cause $\left(C_{1}, \ldots, C_{n}\right)$ then $C_{1}$ is coarser than $C$. To say that $C^{\prime}$ is finer than $C$ simply means that $C$ is coarser than $C^{\prime}$.

We note that, by taking $C=\left(C_{1}, \ldots, C_{n}\right)$ and $C^{\prime}=C_{1}$ (so $C$ is finer than $C^{\prime}$ ), our no-go results (Theorems 4 and 6 and their corollaries) do not follow directly from Proposition 4 of Hofer-Szabó and Rédei ([2004]), which requires a stronger antecedent and delivers a weaker consequent in our setting. ${ }^{26}$ Regarding the antecedent, observe that condition (ii) in the definition of an RCCS is considerably stronger than our 'influence conditions'. As noted in connection with Table 3, our weak influence condition, w-I, requires only that there be one row and one column in which entries differ. Condition (ii) requires that all nine entries in the table differ. Condition (ii) is also stronger than the conjunction of weak influence and ordinal invariance, OI, as can be seen from Tables 3 and 4, which violate (ii) but satisfy both w-I and OI. With respect to strong influence (s-I), our Theorem 5a says that it is possible for composite and component common causes to all screen-off when non-degeneracy (n-D) and strong influence s-I obtain; however, this is impossible under condition (ii). Turning to our claim that Proposition 4 of Hofer-Szabó and Rédei provides a weaker consequent in this setting than our no-go results (Theorems 4 and 6), we note that if the stronger condition (ii) required for an RCCS is assumed, then their Proposition 4 implies that $C_{1}$ fails to be an RCCS for $\left(E_{1}, E_{2}\right)$; this does not, however, entail that $C_{1}$ fails to screen-off $E_{1}$ from $E_{2}$, as the RCCS condition (ii) for $C_{1}$ might fail instead.

Hofer-Szabó and Rédei ([2004]) constructed their concept of an RCCS to generalize a result that Reichenbach (1956) derived. Reichenbach showed that if (i) a dichotomous common cause, $C$, screens-off $E_{1}$ from $E_{2}$ (both of which are dichotomous), and (ii) changing from $C=0$ to $C=1$ has the same directional effect on $E_{1}=1$ that it has on $E_{2}=1$, then it follows that dichotomous

\footnotetext{
${ }^{26}$ Similar obstacles arise if we take $C=C_{1}$ and $C^{\prime}=\left(C_{1}, \ldots, C_{n}\right)$, so that $C$ is coarser than $C^{\prime}$.
} 
effects $E_{1}$ and $E_{2}$ will be positively correlated. Reichenbach's goal was to explain an observed correlation by showing how it can be derived from a set of assumptions about a common cause. Hofer-Szabó and Rédei move from a single dichotomous common cause to a general cause $C$ (which may be individual or composite, and is not required to be dichotomous) and ask how Reichenbach's model can be generalized so as to allow one to deduce the observed correlation of events $E_{1}$ and $E_{2}$. This is the motivation behind viewing their conditions (i) and (ii) as defining an RCCS.

Taking our cue from Hofer-Szabó and Rédei, we note that our weak-influence (w-I), combined with a strengthened form of ordinal invariance, suffices to derive a positive correlation between $E_{1}$ and $E_{2}$ :

\section{Theorem 7}

Suppose that $\left(C_{1}, C_{2}\right)$ screens-off dichotomous effects $E_{1}$ from $E_{2}$, and satisfies n-D, w-I holds for $C_{1}$, and the following strengthening of the OI condition holds:

for all states $g, g^{\prime}$ of $C_{1}$ and $h, h^{\prime}$ of $C_{2}$,

$$
\operatorname{Pr}\left(E_{1} \mid C_{g h}\right)>\operatorname{Pr}\left(E_{1} \mid C_{g^{\prime} h^{\prime}}\right) \Rightarrow \operatorname{Pr}\left(E_{2} \mid C_{g h}\right)>\operatorname{Pr}\left(E_{2} \mid C_{g^{\prime} h^{\prime}}\right) .
$$

Then $E_{1}$ and $E_{2}$ are positively correlated.

See the Appendix for the proof. ${ }^{27}$ Notice that $C_{1}$ and $C_{2}$ can be dichotomous, but they need not be. Notice also that the concept of ordinal invariance used in this theorem is considerably stronger than the concept of ordinal invariance used in Theorem 6, or even in the stronger (symmetric) form required for Corollary 3. In terms of our example based on Tables 3 and 4, OI in our Corollary 3 requires just that there is some row and column in Table 3 , each containing values that are not all the same, that get mirrored or countermirrored in Table 4. The stronger notion of OI that is used in Theorem 7 says that every inequality between pairs of entries in Table 3 must get reproduced in Table 4.

\section{Determinism and Screening-Off: Disanalogy and Analogy}

It is tempting to think that there is an analogy between screening-off and determinism, one that ought to guide our view of how screening-off by a composite cause is related to screening-off by a constituent cause. Consider the following thought about determinism:

D-1: If $\operatorname{Pr}(E \mid C)=1$, then $\operatorname{Pr}(E \mid C \& X)=1$, for any proposition $X$, provided that the second probability is well-defined.

27 Theorem 7 applies to settings in which $\left(C_{1}, C_{2}\right)$ do not form an RCCS for $E_{1}, E_{2}$, and so is not a direct corollary of Proposition 2 of Hofer-Szabó and Rédei ([2004]). Theorem 7 can also be extended to allow $E_{1}$ and $E_{2}$ to be non-dichotomous. 
As noted at the outset, this proposition is true because probabilities of 0 and 1 are sticky. Reasoning by analogy, one might conjecture that screening-off also is sticky:

SO-1: If $C$ screens-off $E_{1}$ from $E_{2}$, then $C \& X$ also screens-off $E_{1}$ from $E_{2}$, for any proposition $X$, provided that $\operatorname{Pr}\left(E_{i} \mid C \& X\right)$ is well-defined.

Another fact about determinism is that the converse of D-1 is false. What is true is that

D-2: If $\operatorname{Pr}(E \mid C \& X)=1$, this leaves open whether $\operatorname{Pr}(E \mid C)=1$,

and so the analogy with determinism may further suggest that

SO-2: If ( $C \& X)$ screens-off $E_{1}$ from $E_{2}$, this leaves open whether $C$ also screens-off $E_{1}$ from $E_{2}$.

This analogy between determinism and screening-off goes awry; D-1 and D-2 are true, but SO-1 is false and SO-2, though true, is misleading. If a less complete cause screens-off, it does not follow that a more complete cause also screens-off (Theorem 5b). ${ }^{28}$ If a more complete cause screens-off, this often does not leave open whether a less complete cause will do so (Corollary 3).

There is a third formal analogy between determinism and screening-off that we should consider. Reichenbach ([1965], pp. 2-3) describes, but does not endorse, 'a law of causality' that says that the probability that an event, $E$, will occur will asymptote to 1 or to 0 as one conditionalizes on more and more complete sets of causes. The suggestion is that ever more inclusive sets of common causes will increase the degree to which those common causes screen-off one effect from the other. To make this idea about monotonicity precise, we need to define what 'degree of screening-off' means. A natural choice is to use the notion of mutual information (Cover and Thomas [1991]). Given a cause, $C$, and two effects, $E_{1}$ and $E_{2}$, the conditional mutual information $I\left(E_{1} ; E_{2} \mid C\right)$ is non-negative, and it is zero precisely when $C$ screens-off $E_{1}$ from $E_{2}$. Understood in this way, the monotonicity thesis is false, as one can see from our Theorem $5 \mathrm{~b} .^{29}$

Although the formal analogy between screening-off and determinism is misguided, there is an epistemic parallel that is worth pondering. Once upon a time, determinism had the status of an a priori principle: if a causal model fails to be deterministic, then it must be incomplete. Stated in this way,

28 The falsehood of SO-1 can be seen by considering the case in which $C$ is a tautology and $J$ is a joint effect of $E_{1}$ and $E_{2}$. If $E_{1}$ and $E_{2}$ are unconditionally independent, a tautology will screen-off $E_{1}$ from $E_{2}$. However, $J$ will render the two events conditionally dependent.

29 Note, however, that if we replace the notion of screening-off with the stronger notion of $d$-separation, then monotonicity is restored; in particular, if a less complete cause $d$-separates two effects, then a more complete cause will also. 
determinism is a metaphysical thesis, but there is a long tradition of regarding it as a 'regulative ideal', the claim being that inquiry benefits from assuming determinism, whether or not determinism is true in fact. Kantians often incline to something stronger-that determinism is a necessary presupposition of rational inquiry. All this changed with the advent of the quantum theory. Not that we now know that determinism is false (Earman [2005]). The point is that we no longer regard determinism as an indispensable assumption. If quantum mechanics or some probabilistic successor theory, is true, we have to acknowledge the possibility that this theory is not just true but causally complete. Of course scientists still need to ponder whether there are hidden causes, but an alertness to the possibility that our models may be causally incomplete in no way requires a commitment to determinism.

A similar dynamic is now underway with respect to the intermediate link, (IL) and the common cause (CC) principles, but it has not gone as far as it should. As noted at the outset, quantum mechanics has led some to doubt CC, but those doubts have rarely reached into other parts of science. With respect to IL, this assumption rarely gets challenged, either inside of quantum mechanics or out. CC and IL, as noted earlier, are consequences of the causal Markov principle. That principle characterizes what will be true in a model that is 'suitable', but the principle plays a role in the causal modelling literature that goes beyond this. For example, Spirtes et al. ([1993/2000]) (see also Verma and Pearl [1990], [1992]; Cooper and Herskovits [1992]) develop algorithms for using probabilistic facts to discover the causal relationships that obtain among a set of variables; these algorithms are derived in part by assuming the causal Markov condition. Paying the cost of assuming the causal Markov condition buys you the benefit of being assured that the model you say is best really is best.

This raises the question of what one should do if CC and IL (and the causal Markov condition that entails both of them) are set to one side. Must causal inquiry grind to a halt? Not at all. Various models can be tested against each other using frequency data; it is frequency data, not a probability distribution over causal variables, that constitute our observations. Some of these models may assume screening-off whereas others do not, and these models may differ in how well they fit the data at hand. Testing models for their fit to data does not require any assumption about what properties a causally complete model must have. But there is more to testing models than checking for fit. In general, a model that assumes screening-off will have fewer adjustable parameters than a model that is otherwise similar but which denies screening-off or leaves open whether screening-off obtains. This matters to model selection criteria like AIC (the Akaike Information Criterion) that take account of both parsimony and fit-to-data (see Forster and Sober [1994] and Sober [2008] for discussion). 
However, the greater parsimoniousness of models that assume screening-off is not a reason to ignore models that do not commit to that assumption. ${ }^{30}$

It may be suggested that scientists should assume the CMC (and the CC and IL principles that it entails) until a concrete empirical example is discovered that refutes it. Surely it would be a mistake to reject the principle just because it is logically possible that it is false. In reply, we note that we are not suggesting that the $\mathrm{CMC}$ is false. Rather, we are questioning the idea that it should be assumed. Just as is true of determinism, it is a mistake to think that the principle should be assumed innocent until observations prove that it is guilty.

Our Theorem 6 says that if the common causes mentioned in model $M$ screen-off their effects from each other, then a supermodel that subsumes those causes will do the same only if that supermodel violates faithfulness. Believers in faithfulness can then conclude that $M$ must be causally complete just because it induces screening-off. We recommend agnosticism with respect to faithfulness just as we do with respect to CMC. It may be a reasonable rule of thumb, but we know of no compelling reason to think it must be true without exception. Scientific inquiry has no need of such a hypothesis. We should note that we are not impressed by the fact that violations of faithfulness involve set-ups that have measure zero on the standard measure. The same is true of screening-off, but that is no reason to reject the CMC.

Even if one abandons an a priori allegiance to IL and CC, the thought may linger that there is abundant empirical evidence that both these principles are true. ${ }^{31}$ Consider the Mendelian example of a parental pair that screens-off one offspring's genotype from the others. If Mendelism is empirically successful, doesn't that provide evidence that $\mathrm{CC}$ is true? Our no-go results (Theorem 6 and Corollary 3 ) constitute a reason to be sceptical. The fact that $C$ screens-off $E_{1}$ from $E_{2}$ does not provide evidence that a causally complete model that includes $C$ will also screen-off. What is true is that if $C$ screens-off and is causal complete, then this is 'instance confirmation' of the general CC principle. But what if $C$ is causally incomplete? What does the fact that $C$ screens-off say about a causally complete model $M$ that subsumes $C$ ? The answer (according to Corollary 3 ) is that the composite cause described in $M$ must fail to screen-off $E_{1}$ from $E_{2}$ if the component causes in $M$ obey non-degeneracy, the weak influence condition (w-I), and ordinal invariance. If the components

30 A similar argument can be constructed concerning the requirement that a causal model should obey the constraint of faithfulness, but we lack the space to develop that argument here.

31 Spirtes et al. ([1993/2000], p. 38) offer two defences of the causal Markov condition: 'the basis for the CMC is first, that it is necessarily true of populations of structurally alike pseudoindeterministic systems whose exogenous variables are distributed independently, and second, it is supported by almost all of our experience with systems that can be put through repetitive processes and whose fundamental propensities can be tested'. They make the same two-part argument on page 32. Our disagreement is with the second reason; we will not discuss the first. 
obey non-degeneracy and weak influence, but violate ordinal invariance, then it is possible for the composite cause in $M$ to screen-off, but for this to be true, various probabilities must have their values set just so. ${ }^{32} \mathrm{~A}$ similar point applies if the weak-influence condition is violated by the component causes in $M .^{33}$ Of course, if we think that $C$ is true but incomplete, we probably don't know what the other causes are that need to be added to $C$ to obtain a complete causal model. If we don't know what these other causes are, it is hard to see how we can say, one way or the other, whether that complete set of causes will obey ordinal invariance. This is why we think the CC and IL principles are not confirmed by finding causes that screen-off that one thinks may be incomplete.

Our argument concerns the relationship of a cause that is found to screen-off and a superset of causes. This invites the reply that the causes we discover are not just incomplete, but stand in need of replacement. For example, if $C$ is an incomplete common cause that screens-off, perhaps $C$ should be removed and replaced by a set of common causes that fails to include $C$. Maybe CC concerns this replacement set. Perhaps so. But this is no argument for the truth of CC or IL, nor does it show that C's screening-off provides evidence that this replacement set of common causes will do so as well.

This lesson concerning CC and IL applies to any similar principle that says that some suitably specified set of composite common causes (or of intermediate links), must induce screening-off. Corollary 3 shows that finding an empirical example of screening-off (where one has no assurance that the model one has constructed is causally complete) cannot be regarded as evidence for any such principle.

Just as a model that asserts screening-off is more parsimonious than a model that is otherwise similar except that screening-off is not assumed, so a deterministic model is more parsimonious than a model that is otherwise similar except that it leaves open what probabilities causes assign to effects. This modest epistemic point is what gives determinism and screening-off their special status. But acknowledging this does not bring with it a commitment to the truth of determinism or to the truth of IL and CC. ${ }^{34}$

${ }^{32}$ If $M$ and $C$ both screen-off, $M$ will violate faithfulness. This gives fans of faithfulness an additional reason for thinking that $C$ 's screening-off is not evidence that $\mathrm{CC}$ is true.

33 Consider Figure 1 and suppose that $C_{d}$ and $C_{p}$ constitute a complete set of common causes of $E_{1}$ and $E_{2}$. These two causes will violate weak-influence if $C_{p}$ screens-off $C_{d}$.

34 There is another way to argue that parsimony helps justify screening-off principles. In the Principles of Philosophy (Section 39), Descartes ([1644]) states his second law of nature and asks why it is true. The law says that 'all motion is in itself rectilinear'; this means that rectilinear motion will occur if no outside cause impinges. Descartes says this law owes its truth to 'the immutability and simplicity of the operation by which God preserves motion in matter. For he always preserves the motion in the precise form in which it is occurring at the very moment when he preserves it, without taking any account of the motion which was occurring a little while earlier'. 


\section{Funding}

William F. Vilas Trust (to Elliot Sober); Royal Society of New Zealand (to Mike Steel).

\section{Acknowledgements}

We thank Martin Barrett, Jeremy Butterfield, Mehmet Elgin, Malcolm Forster, Roman Frigg, Daniel Hausman, Christopher Hitchcock, Gabor Hofer-Szabó, Matthew Kopec, Richard Lewontin, Miklós Rédei, Richard Scheines, Eric Stencil, Mike Titelbaum, Jos Uffink, Naftali Weinberger, and Karen Zweier for useful discussion. We also thank the anonymous reviewers for some helpful suggestions.

Elliott Sober

Philosophy Department University of Wisconsin Madison, Wisconsin 53706, USA ersober@wisc.edu

Mike Steel Biomathematics Research Centre University of Canterbury, Private Bag 4800 Christchurch, New Zealand mike.steel@canterbury.ac.nz

\section{Appendix: Proofs of Theorems 2-6 and Corollaries 2 and 3}

Proof of Theorem 2 (first claim in part (ii)):

We write $+C_{p}$ and $-\mathrm{C}_{p}$ for events $C_{p}=1$ and $C_{p}=0$, respectively, and use analogous notation for $\pm C_{d}$ and for $\pm E_{i}$. The value of $C_{d}$ is chosen by tossing a fair coin, and the value of $C_{p}$ depends on $C_{d}$ as follows: $\operatorname{Pr}\left(+C_{p} \mid+C_{d}\right)=2 / 3$ and $\operatorname{Pr}\left(+C_{p} \mid-C_{d}\right)=1 / 3$, which gives $\operatorname{Pr}\left(+C_{p}\right)=1 / 2$. For $C_{p}$ to screen-off $E_{1}$ from $E_{2}$ means that:

$$
\operatorname{Pr}\left(+E_{1} \&+E_{2} \mid+C_{p}\right)=\operatorname{Pr}\left(+E_{1} \mid+C_{p}\right) \cdot \operatorname{Pr}\left(+E_{2} \mid+C_{p}\right)
$$

and

$$
\operatorname{Pr}\left(+E_{1} \&+E_{2} \mid-C_{p}\right)=\operatorname{Pr}\left(+E_{1} \mid-C_{p}\right) \cdot \operatorname{Pr}\left(+E_{2} \mid-C_{p}\right),
$$

and it will simplify the calculations to also impose the constraint:

$$
\operatorname{Pr}\left(+E_{1} \&-E_{2} \mid \pm C_{p} \& \pm C_{d}\right)=\operatorname{Pr}\left(-E_{1} \&+E_{2} \mid \pm C_{p} \& \pm C_{d}\right),
$$

so

$$
\operatorname{Pr}\left(+E_{1} \mid \pm C_{p} \& \pm C_{d}\right)=\operatorname{Pr}\left(+E_{2} \mid \pm C_{p} \& \pm C_{d}\right)
$$


We will also insist that no probability (conditional and absolute) equals 0 or 1 and that, for $i=1,2$,

$$
\operatorname{Pr}\left(+E_{i} \mid+C_{p}\right)>\operatorname{Pr}\left(+E_{i}\right) .
$$

Finally, we require that $C_{p}$ does not screen-off $C_{d}$ from $E_{i}(i=1,2)$ for which a sufficient condition is that, for $i=1$ and $i=2$ :

$$
\operatorname{Pr}\left(+E_{i} \mid+C_{p} \&+C_{d}\right)>\operatorname{Pr}\left(+E_{i} \mid+C_{p}\right) .
$$

To satisfy all these conditions, we let (for $x, y \in\{0,1\}$ ):

$$
\begin{aligned}
a_{x y} & :=\operatorname{Pr}\left(C_{d}=x \& C_{p}=y\right) ; \\
p_{x y} & :=\operatorname{Pr}\left(+E_{1} \mid C_{d}=x \& C_{p}=y\right)=\operatorname{Pr}\left(+E_{2} \mid C_{d}=x \& C_{p}=y\right) ; \text { and } \\
q_{x y} & :=\operatorname{Pr}\left(+E_{1} \&+E_{2} \mid C_{d}=x \& C_{p}=y\right),
\end{aligned}
$$

where the second equality is by exchangeability. Note that $a_{00}=a_{11}=1 / 3, a_{01}$ $=a_{10}=1 / 6$. We now specify $p_{x y}$ and $q_{x y}$ values:

$$
\begin{aligned}
& p_{11}=\frac{1}{2}, p_{01}=p_{10}=\frac{1}{3}, p_{00}=\frac{1}{4} ; \\
& q_{11}=\frac{5}{18}, q_{01}=q_{10}=\frac{1}{27}, q_{00}=\frac{7}{72} .
\end{aligned}
$$

To check Equation (A.1) we have

$$
\begin{aligned}
& \operatorname{Pr}\left(+E_{1} \&+E_{2} \mid+C_{p}\right)= \\
& \operatorname{Pr}\left(+E_{1} \& E_{2} \mid+C_{d} \&+C_{p}\right) \cdot \operatorname{Pr}\left(+C_{d} \mid+C_{p}\right) \\
& \quad+\operatorname{Pr}\left(+E_{1} \&+E_{2} \mid-C_{d} \&+C_{p}\right) \cdot \operatorname{Pr}\left(-C_{d} \mid+C_{p}\right),
\end{aligned}
$$

and, by Bayes' formula, $\operatorname{Pr}\left(+C_{d} \mid+C_{p}\right)=\operatorname{Pr}\left(+C_{p} \mid+C_{d}\right) \cdot \operatorname{Pr}\left(+C_{d}\right) / \operatorname{Pr}\left(+C_{p}\right)$ $=2 / 3$. Thus,

$$
\operatorname{Pr}\left(+E_{1} \&+E_{2} \mid+C_{p}\right)=\frac{2}{3} q_{11}+\frac{1}{3} q_{01},
$$

while, for $i=1,2$ :

$$
\begin{aligned}
\operatorname{Pr}\left(+E_{i} \mid+C_{p}\right)= & \operatorname{Pr}\left(+E_{i} \mid+C_{d} \&+C_{p}\right) \cdot \operatorname{Pr}\left(+C_{d} \mid+C_{p}\right) \\
& +\operatorname{Pr}\left(+E_{i} \mid-C_{d} \&+C_{p}\right) \cdot \operatorname{Pr}\left(-C_{d} \mid+C_{p}\right) .
\end{aligned}
$$

Thus,

$$
\operatorname{Pr}\left(+E_{i} \mid+C_{p}\right)=\frac{2}{3} p_{11}+\frac{1}{3} p_{01} .
$$

Equation (A.1) now follows from Equations (A.7) and (A.8) since $\frac{2}{3} q_{11}+\frac{1}{3} q_{01}=\left(\frac{2}{3} p_{11}+\frac{1}{3} p_{01}\right)^{2}$. Similarly, Equation (A.2), corresponding to the condition $\frac{1}{3} q_{10}+\frac{2}{3} q_{00}=\left(\frac{2}{3} p_{00}+\frac{1}{3} p_{01}\right),{ }^{2}$ also holds. Regarding inequality (A.5), we have $\operatorname{Pr}\left(+E_{i} \mid+C_{p}\right)=\frac{2}{3} p_{11}+\frac{1}{3} p_{01}$ from Equation (A.8). Using a similar expansion for $\operatorname{Pr}\left(+E_{i}\right)$, inequality (A.5) becomes equivalent to 
$\frac{2}{3} p_{11}+\frac{1}{3} p_{01}>\frac{1}{3} p_{11}+\frac{1}{6} p_{01}+\frac{1}{6} p_{10}+\frac{1}{3} p_{00}$, which holds; whereas inequality (A.6) corresponds to $p_{11}>\frac{2}{3} p_{11}+\frac{1}{3} p_{01}$, which also holds.

It remains to show that there exists a probability distribution for the 16 values $\left( \pm E_{1}, \pm E_{2}, \pm C_{p}, \pm C_{d}\right)$ that provides the $p_{x y}, q_{x y}$, and $a_{x y}$ values described. Actually, we have no choice as to what this distribution must be, since from $p_{x y}$ and $q_{x y}$ we can determine for each $x, y$, the four values $\operatorname{Pr}\left( \pm E_{1} \& \pm E_{2} \mid C_{d}=x, C_{p}=y\right)$. In particular,

$$
\begin{gathered}
\operatorname{Pr}\left(+E_{1} \&+E_{2} \mid C_{d}=x \& C_{p}=y\right)=q_{x y}, \\
\operatorname{Pr}\left(+E_{1} \&-E_{2} \mid C_{d}=x \& C_{p}=y\right)=p_{x y}-q_{x y}=\operatorname{Pr}\left(-E_{1} \&+E_{2} \mid C_{d}=x \& C_{p}=y\right), \\
\operatorname{Pr}\left(-E_{1} \&-E_{2} \mid C_{d}=x \& C_{p}=y\right)=1+q_{x y}-2 p_{x y} .
\end{gathered}
$$

It is easily checked that these four values (for each $x, y$ ) are non-negative, and sum to 1 ; from this we obtain the unique joint probability distribution for the 16 values $\left( \pm E_{1}, \pm E_{2}, \pm C_{p}, \pm C_{d}\right)$ since, for each $e_{1}, e_{2} \in$ $\{0,1\}$, we have:

$$
\begin{aligned}
\operatorname{Pr}\left(E_{1}\right. & \left.=e_{1} \& E_{2}=e_{2} \& C_{d}=x \& C_{p}=y\right) \\
& =\operatorname{Pr}\left(E_{1}=e_{1} \& E_{2}=e_{2} \mid C_{d}=x \& C_{d}=y\right) \cdot a_{x y} .
\end{aligned}
$$

This completes the proof. We note that, in our construction, $C_{d}$ also screensoff $E_{1}$ from $E_{2}$ (as does $C_{p}$ ), yet the conjunctive pair fails to, since

$$
\begin{aligned}
\operatorname{Pr}\left(+E_{1} \&+E_{2} \mid+C_{d} \&+C_{p}\right) & =q_{11} \neq p_{11}^{2} \\
& =\operatorname{Pr}\left(+E_{1} \mid+C_{d} \&+C_{p}\right) \cdot \operatorname{Pr}\left(+E_{2} \mid+C_{d} \&+C_{p}\right) .
\end{aligned}
$$

\section{Proof of Theorem 3a}

Suppose that $C^{\prime}$ satisfies both n-D and w-I. Write $C^{\prime}=(C, D)$ where $D$ is the additional event(s) in $C^{\prime}$ that are not in $C$ (note that both $C$ and $D$ can be composite events). Then the w-I assumption implies the existence of a state $c$ of $C$ and states $d, d^{\prime}$ of $D$ for which:

$$
\operatorname{Pr}(E \mid C=c \& D=d) \neq \operatorname{Pr}\left(E \mid C=c \& D=d^{\prime}\right) .
$$

Now, since $C$ is deterministic, one of the values in this inequality is 0 and the other is 1; without loss of generality we may suppose that $\operatorname{Pr}(E \mid C=c \& D=d)=0$. Consider now $\operatorname{Pr}(E \mid C=c)$. By the law of total probability, this can be written:

$$
\operatorname{Pr}(E \mid C=c)=\sum_{x} \operatorname{Pr}(E \mid C=c \& D=x) \operatorname{Pr}(D=x \mid C=c),
$$

where the summation is over all states, $x$, that $D$ can take. The n-D assumption implies that $\operatorname{Pr}(D=x \mid C=c)>0$ for all states $x$ of $D$. 
Now, since $\operatorname{Pr}(E \mid C=c \& D=d)=0$, Equation (A.9) implies that $\operatorname{Pr}(E \mid C=c)$ equals:

$$
\begin{aligned}
\sum_{x \neq d} \operatorname{Pr}(E \mid C=c \& D=x) \operatorname{Pr}(D=x \mid C=c) & \leq \sum_{x \neq d} \operatorname{Pr}(D=x \mid C=c) \\
& =1-\operatorname{Pr}(D=d \mid C=c)
\end{aligned}
$$

and so, by n-D, $\operatorname{Pr}(E \mid C=c)<1$, which implies (since $C$ is deterministic) that:

$$
\operatorname{Pr}(E \mid C=c)=0 .
$$

But Equation (A.9) and n-D also implies that:

$\operatorname{Pr}(E \mid C=c) \geq \operatorname{Pr}\left(E \mid C=c \& D=d^{\prime}\right) \operatorname{Pr}\left(D=d^{\prime} \mid C=c\right)=\operatorname{Pr}\left(D=d^{\prime} \mid C=c\right)>0$, which contradicts Equation (A.10). This contradiction ensure that $C^{\prime}$ cannot satisfy both n-D and w-I, as claimed.

\section{Proof of Theorem 3b}

If $C$ has $m$ states and screens-off $E_{1}$ from $E_{2}$ then:

$$
\operatorname{Pr}\left(E_{1}=i \& E_{2}=j\right)=\sum_{g=1}^{m} \operatorname{Pr}\left(E_{1}=i \mid C=g\right) \operatorname{Pr}\left(E_{2}=j \mid C=g\right) \operatorname{Pr}(C=g) .
$$

Let $v_{g}^{1}$ and $v_{g}^{2}$ be the row vectors of dimension $k$ whose $i$-th coordinate is $\operatorname{Pr}\left(E_{1}=i \mid \mathrm{C}=g\right)$ and $\operatorname{Pr}\left(E_{2}=i \mid \mathrm{C}=g\right)$, respectively, and let $p_{g}=\operatorname{Pr}(C=g)$ then:

$$
P=\sum_{g=1}^{m} p_{g} \cdot v_{g}^{1}\left(v_{g}^{2}\right)^{\mathrm{T}}
$$

where $\mathrm{T}$ denotes transpose. Now, each matrix $p_{g} \cdot v_{g}^{1}\left(v_{g}^{2}\right)^{\mathrm{T}}$ has rank 1 . By the subadditivity property of matrix $\operatorname{rank}, \operatorname{rank}(P) \leq(1+1+\cdots+1)=m$. Finally, $\operatorname{det}(P) \neq 0$ implies $\operatorname{rank}(P)=k$ so $m \geq k$ as claimed.

\section{Proof of Theorem 4}

Assume that n-D holds, and that $\left(C_{1}, C_{2}\right)$ screens-off $E_{1}$ from $E_{2}$, that $C_{1}$ does so also, and that $C_{2}$ is dichotomous. We will show that the strong influence property (s-I) fails for any arbitrarily selected state of $C_{1}$, which we may take as state 1 . Consider the conditional probabilities $\operatorname{Pr}\left(E_{i j} \mid C_{g h}\right)$ (which exist by $n-D)$ defined as follows:

$$
\begin{aligned}
& x_{1}=\operatorname{Pr}\left(E_{11} \mid C_{11}\right), x_{2}=\operatorname{Pr}\left(E_{11} \mid C_{10}\right) ; \\
& y_{1}=\operatorname{Pr}\left(E_{10} \mid C_{11}\right), y_{2}=\operatorname{Pr}\left(E_{10} \mid C_{10}\right) ; \\
& y_{1}^{\prime}=\operatorname{Pr}\left(E_{01} \mid C_{11}\right), y_{2}^{\prime}=\operatorname{Pr}\left(E_{01} \mid C_{10}\right) .
\end{aligned}
$$

For $i=1,2$, let $S_{i}=x_{i}+y_{i}$ and $S_{i}^{\prime}=x_{i}+y_{i}^{\prime}$. Thus,

$$
S_{1}=\operatorname{Pr}\left(E_{1}=1 \mid C_{11}\right), \quad S_{1}^{\prime}=\operatorname{Pr}\left(E_{2}=1 \mid C_{11}\right),
$$


and

$$
S_{2}=\operatorname{Pr}\left(E_{1}=1 \mid C_{10}\right), S_{2}^{\prime}=\operatorname{Pr}\left(E_{2}=1 \mid C_{10}\right) .
$$

The requirement that $E_{1}=1$ and $E_{2}=1$ are conditionally independent given $C_{11}$ means that:

$$
x_{1}=\left(x_{1}+y_{1}\right) \cdot\left(x_{1}+y_{1}^{\prime}\right)=S_{1} S_{1}^{\prime} .
$$

Similarly, conditional independence of $E_{1}$ and $E_{2}$ given $C_{10}$ means that:

$$
x_{2}=S_{2} S_{2}^{\prime} .
$$

We now turn to the consequence of $C_{1}=1$ screening off $E_{1}$ from $E_{2}$. This gives:

$$
x_{1} c+x_{2}(1-c)=\left(S_{1} c+S_{2}(1-c)\right) \cdot\left(S_{1}^{\prime} c+S_{2}^{\prime}(1-c)\right),
$$

where $c=\operatorname{Pr}\left(C_{2}=1 \mid C_{1}=1\right)$. Expanding Equation (A.13), simplifying, and dividing both sides by $c(1-c)$ (noting that $0<c<1$, by n-D) and invoking Equations (A.11) and (A.12) to replace $x_{1}$ and $x_{2}$ by $S_{1} S_{1}^{\prime}$ and $S_{2} S_{2}^{\prime}$, respectively, gives

$$
\left(S_{1}-S_{2}\right)\left(S_{1}^{\prime}-S_{2}^{\prime}\right)=0
$$

from which we conclude that

$$
S_{1}=S_{2} \text { or } S_{1}^{\prime}=S_{2}^{\prime} \text {. }
$$

The first of these equalities states that $\operatorname{Pr}\left(E_{1}=1 \mid C_{11}\right)=\operatorname{Pr}\left(E_{1}=1 \mid C_{10}\right)$, which violates the strong influence condition (s-I); the second states that $\operatorname{Pr}\left(E_{2}=1 \mid C_{11}\right)=\operatorname{Pr}\left(E_{2}=1 \mid C_{10}\right)$, which also violates s-I. An analogous argument applies if $C_{2}$ screens-off $E_{1}$ from $E_{2}$. This completes the proof of the first part of the Theorem; the second part follows immediately by two applications of the first part.

\section{Proof of Corollary 2}

Let $C_{1}^{\prime}=\left(C_{1}, C_{2}, \ldots, C_{k-1}\right)$ and $C_{2}^{\prime}=C_{k}$. Then $C_{1}^{\prime}, C_{2}^{\prime}, E_{1}$, and $E_{2}$ also satisfy conditions n-D and s-I, and $C_{2}^{\prime}$ is dichotomous. So Theorem 4 implies that either $\left(C_{1}^{\prime}, C_{2}^{\prime}\right)$ or $C_{1}^{\prime}$ fails to screen-off $E_{1}$ from $E_{2}$. But the first alternative is excluded since $\left(C_{1}^{\prime}, C_{2}^{\prime}\right)=\left(C_{1}, C_{2}, \ldots, C_{k}\right)$, which we are assuming screens-off $E_{1}$ from $E_{2}$.

\section{Proof of Theorem 6}

Suppose that $\left(C_{1}, C_{2}\right)$ screens-off $E_{1}$ from $E_{2}$. For the fixed states $g$ and $j$ in the statement of Theorem 6 , and any $h \in H$, let

$$
\begin{gathered}
x_{g h}:=\operatorname{Pr}\left(E_{1}=j \& E_{2}=j^{\prime} \mid C_{g h}\right) \\
y_{g h}:=\sum_{k \in J_{2}-\left\{j^{\prime}\right\}} \operatorname{Pr}\left(E_{1}=j \& E_{2}=k \mid C_{g h}\right)
\end{gathered}
$$


and

$$
y_{g h}^{\prime}:=\sum_{k \in J_{1}-\{j\}} \operatorname{Pr}\left(E_{1}=k \& E_{2}=j^{\prime} \mid C_{g h}\right)
$$

In addition, let

$$
S_{g h}:=x_{g h}+y_{g h} ; S_{g h}^{\prime}=x_{g h}+y_{g h}^{\prime} ; \text { and } c_{g h}:=\operatorname{Pr}\left(C_{2}=h \mid C_{1}=g\right) .
$$

By definition:

$$
S_{g h}=\operatorname{Pr}\left(E_{1}=j \mid C_{g h}\right) ; S_{g h}^{\prime}=\operatorname{Pr}\left(E_{2}=j^{\prime} \mid C_{g h}\right),
$$

and

$$
\operatorname{Pr}\left(E_{1}=j \& E_{2}=j^{\prime} \mid C_{1}=g\right)=\sum_{h \in H} x_{g h} \cdot c_{g h}
$$

The assumption that $C_{g h}$ screens-off $E_{1}$ from $E_{2}$ for all $g, h$ gives:

$$
x_{g h}=S_{g h} \cdot S_{g h}^{\prime},
$$

The condition that $C_{1}$ screens-off $E_{1}=j$ from $E_{2}=j^{\prime}$ is equivalent to the condition that for all $g \in G$ :

$$
\operatorname{Pr}\left(E_{1}=j \& E_{2}=j^{\prime} \mid C_{1}=g\right)=\operatorname{Pr}\left(E_{1}=j \mid C_{1}=g\right) \operatorname{Pr}\left(E_{2}=j^{\prime} \mid C_{1}=g\right),
$$

which, in view of the previous equations, becomes:

$$
\sum_{h \in H} x_{g h} \cdot c_{g h}=\left(\sum_{h \in H} S_{g h} \cdot c_{g h}\right) \cdot\left(\sum_{h \in H} S_{g h}^{\prime} \cdot c_{g h}\right) .
$$

Applying Equation (A.15), this is further equivalent to the condition:

$$
\sum_{h \in H} S_{g h} \cdot S_{g h}^{\prime} \cdot c_{g h}=\left(\sum_{h \in H} S_{g h} \cdot c_{g h}\right) \cdot\left(\sum_{h \in H} S_{g h}^{\prime} \cdot c_{g h}\right) .
$$

We now invoke a standard trick used to establish correlation inequalities like the Chebyshev order inequality (see, for example Steele [2004]). Consider the double sum:

$$
\Delta_{g}:=\sum_{h \in H, k \in H}\left(S_{g h}-S_{g k}\right) \cdot\left(S_{g h}^{\prime}-S_{g k}^{\prime}\right) \cdot c_{g h} \cdot c_{g k} .
$$

Expanding out $\Delta_{g}$ as

$$
\Delta_{g}=2 \sum_{h \in H} S_{g h} \cdot S_{g h}^{\prime} \cdot c_{g h} \cdot \sum_{k \in H} c_{g k}-\left(\sum_{h \in H} S_{g h} \cdot c_{g h}\right) \cdot\left(\sum_{k \in H} S_{g k}^{\prime} \cdot c_{g k}\right)
$$

and, noting that $\sum_{k \in H} c_{g k}=1$, it follows that $\Delta_{g}$ is zero for all $g \in G$ if and only if $C_{1}$ screens-off $E_{1}=j$ from $E_{2}=j^{\prime}$. 
Now, by ordinal invariance, all the summation terms in this expression for $\Delta_{g}$ given by Equation (A.17) have the same sign. Moreover, since $C_{2}$ weakly influences $E_{1}$ with revelatory state $g$ for $C_{1}$, and influenced state $j$ for $E_{1}$, and since $c_{g h}, c_{g k} \neq 0$ by n-D, at least one term in Equation (A.17) non-zero and so the sum is non-zero. Since $\Delta_{g} \neq 0$, this implies that $C_{1}$ fails to screen-off $E_{1}=j$ from $E_{2}=j^{\prime}$.

\section{Proof of Theorem 5a}

The previous proof (of Theorem 6) shows that if $\left(C_{1}, C_{2}\right)$ screens off $E_{1}$ from $E_{2}$ (both dichotomous), then $C_{1}$ does also, provided that $\Delta_{g}=0$ for each of the states $g=1,2,3$ for $C_{1}$, where $\Delta_{g}$ is defined by (18). Since the causes in the example associated with Theorem $5 \mathrm{a}$ are independent, we also have $c_{g h}=\operatorname{Pr}\left(C_{2}=h\right)$, which we will denote by $c_{h}$. The equations $\Delta_{g}=0$ for the three values of $g$ are exactly the same equation, which can be rescaled and written as:

$$
2 c_{1} c_{2}+2 c_{1} c_{3}-c_{2} c_{3}=0 .
$$

Noting that $c_{1}+c_{2}+c_{3}=1$ we can write this as $2 \mathrm{c}_{1}\left(1-\mathrm{c}_{1}\right)=c_{2} \mathrm{c}_{3}$, which has many solutions, including one in which $c_{2}=c_{3}$ in which case $c_{1}=1 / 9, c_{2}=$ $c_{3}=4 / 9$.

\section{Proof of Corollary 3}

Apply Theorem 6, by considering two causes $D_{1}$ and $D_{2}$ for $E_{1}, E_{2}$, where $D_{1}=$ $C_{i}$, and $D_{2}$ is the conjunction of the remaining $n-1$ causes. Observe that the assumption of n-D for $C_{1}, C_{2}, \ldots, C_{n}$ implies that n-D also holds for $D_{1}, D_{2}$. Moreover, condition ( $\mathrm{I} \mid D_{1}$ ) applies if we take $j=j_{i}, j^{\prime}=j_{i}^{\prime}$ and $g=g_{i}$, and $E_{1}$ and $E_{2}$ satisfy ordinal invariance relative to $\left(D_{1}, D_{2}, g, j, j^{\prime}\right)$. Now, if $\left(C_{1}\right.$, $\left.C_{2}, \ldots, C_{n}\right)$ screens-off $E_{1}$ from $E_{2}$ then $\left(D_{1}, D_{2}\right)$ does also, and so, by Theorem $6, D_{1}=C_{i}$ fails to screen-off $E_{1}$ from $E_{2}$. Since this holds for all values of $i$, this establishes Corollary 3 .

\section{Proof of Theorem 7}

For states $g$ and $h$ for $C_{1}$ and $C_{2}$, respectively, let $C_{g h}$ denote the event $C_{1}=g \& C_{2}=h$, and let $u_{g h}:=\operatorname{Pr}\left(E_{1} \mid C_{g h}\right), v_{g h}:=\operatorname{Pr}\left(E_{2} \mid C_{g h}\right)$, and $w_{g h}:=\operatorname{Pr}\left(C_{g h}\right)$. Then

$$
\operatorname{Pr}\left(E_{1} \& E_{2}\right)=\sum_{g, h} \operatorname{Pr}\left(E_{1} \& E_{2} \mid C_{g h}\right) \operatorname{Pr}\left(C_{g h}\right)=\sum_{g, h} u_{g h} v_{g h} w_{g h},
$$

(the second equality holds by the assumption that $\left(C_{1}, C_{2}\right)$ screens-off $E_{1}$ from $\left.E_{2}\right)$. Similarly,

$$
\operatorname{Pr}\left(E_{1}\right)=\sum_{g, h} u_{g h} w_{g h} \text { and } \operatorname{Pr}\left(E_{2}\right)=\sum_{g, h} v_{g h} w_{g h} .
$$


Thus, if we let $\delta:=\operatorname{Pr}\left(E_{1} \& E_{2}\right)-\operatorname{Pr}\left(E_{1}\right) \operatorname{Pr}\left(E_{2}\right)$ denote the (unscaled) correlation between $E_{1}$ and $E_{2}$, then:

$$
\delta=\sum_{g, h} u_{g h} v_{g h} w_{g h}-\sum_{g, h} u_{g h} w_{g h} \cdot \sum_{g, h} v_{g h} w_{g h} .
$$

Consider the quantity:

$$
\sum_{g, g^{\prime}} \sum_{h, h^{\prime}}\left(u_{g h}-u_{g^{\prime} h^{\prime}}\right) \cdot\left(v_{g h}-v_{g^{\prime} h^{\prime}}\right) w_{g h} w_{g^{\prime} h^{\prime}}
$$

This sum is non-negative, due to the the stronger form of OI in Theorem 7; moreover, it is non-zero by w-I and n-D, and so it is strictly positive. However, expansion of this expression and gathering like terms shows that it is just $2 \delta$. Thus $\delta>0$, as claimed.

\section{References}

Boutilier, C., Friedman, N., Goldszmidt, M. and Koller, D. [1996]: 'Context-Specific Independence in Bayesian Networks', Proceedings of the Twelfth Conference on Uncertainty in Artificial Intelligence, San Francisco CA, USA: Morgan Kaufmann Publishers Inc., pp. 115-23.

Cartwright, N. [2002]: 'Against Modularity, the Causal Markov Condition, and Any Link between the Two: Comments on Hausman and Woodward', British Journal for the Philosophy of Science, 53, pp. 411-53.

Cartwright, N. [2007]: Hunting Causes and Using Them, Cambridge: Cambridge University Press.

Cooper, G. F. and Herkovits, E. [1992]: 'A Bayesian Method for the Induction of Probabilistic Networks from Data', Machine Learning, 9, pp. 309-47.

Cover, T. M. and Thomas, J. A. [1991]: Elements of Information Theory, New York: Wiley.

Descartes, R. [1644/2010]: Principles of Philosophy, in J. Veitch (ed. and trans.), The Method, Meditations, and Selections from the Principles of Descartes, Montana: Kessinger Publishing.

Earman, J. [2004]: 'Determinism: What We Have Learned and What We Still Don't Know', in J. Campbell, M. O'Rourke and D. Shier (eds), Freedom and Determinism, Topics in Contemporary Philosophy Series, Volume II, Cambridge, MA: MIT Press, pp. 21-46.

Forster, M. R. and Sober, E. [1994]: 'How to Tell When Simpler, More Unified, or Less Ad Hoc Theories Will Provide More Accurate Predictions', British Journal for the Philosophy of Science, 45, pp. 1-36.

Hitchcock, C. [2010]: 'Probabilistic Causation', in E. N. Zalta (ed.), The Stanford Encyclopedia of Philosophy, <http://plato.stanford.edu/archives/win2011/ entries/causation-probabilistic/>.

Hofer-Szabó, G. and Rédei, M. [2004]: 'Reichenbachian common cause systems', International Journal of Theoretical Physics, 43, pp. 1819-26. 
Pearl, J. [2009]: Causality: Models, Reasoning, and Inference, New York: Cambridge University Press.

Reichenbach, H. [1956]: The Direction of Time, Berkeley, CA: University of California Press.

Reichenbach, H. [1965]: Philosophic Foundations of Quantum Mechanics, Berkeley, CA: University of California Press.

Sober, E. [1988]: Reconstructing the Past: Parsimony, Evidence, and Inference, Cambridge: MIT Press.

Sober, E. [2001]: 'Venetian Sea Levels, British Bread Prices, and the Principle of the Common Cause', British Journal for the Philosophy of Science, 52, pp. 331-46.

Sober, E. [2008]: Evidence and Evolution: The Logic Behind the Science, Cambridge: Cambridge University Press.

Spirtes, P., Glymour, C. and Scheines, R. [2000]: Causation, Prediction, and Search, Cambridge, MA: MIT Press.

Steele, J. M. [2004]: The Cauchy-Schwarz Master Class: An Introduction the Art of Mathematical Inequalities, Cambridge: Cambridge University Press.

Uffink, J. [1999]: 'The Principle of the Common Cause Faces the Bernstein Paradox', Philosophy of Science, 66, pp. S512-25.

Van Fraassen, B. [1982]: 'The Charybdis of Realism: Epistemological Implications of Bell's Inequality', Synthese, 52, pp. 25-38.

Verma, T. S. and Pearl, J. [1990]: 'Equivalence and Synthesis of Causal Models', in P. P. Bonissone, L. N. Kanal, J. F. Lemmer and M. Henrion (eds), Proceedings of the Sixth Annual Conference on Uncertainty in Artificial Intelligence 1991, New York: Elsevier, pp. 255-70.

Verma, T. S. and Pearl, J. [1992]: 'An Algorithm for Deciding if a Set of Observed Independencies Has a Causal Explanation', Proceedings of the Eighth Conference on Uncertainty in Artificial Intelligence, San Francisco: Morgan Kaufmann Publishers, pp. 323-30.

Woodward, J. [2003]: Making Things Happen, Oxford: Oxford University Press.

Zhang, J. and Spirtes, P. [2008]: 'Detection of Unfaithfulness and Robust Causal Inference', Minds and Machines, 18, pp. 239-71. 